TRANSACTIONS OF THE

AMERICAN MATHEMATICAL SOCIETY

Volume 359, Number 1, January 2007, Pages 249-274

S 0002-9947(06)03898-0

Article electronically published on July 20, 2006

\title{
HESSIAN NILPOTENT POLYNOMIALS AND THE JACOBIAN CONJECTURE
}

\author{
WENHUA ZHAO
}

\begin{abstract}
Let $z=\left(z_{1}, \cdots, z_{n}\right)$ and let $\Delta=\sum_{i=1}^{n} \frac{\partial^{2}}{\partial z_{i}^{2}}$ be the Laplace operator. The main goal of the paper is to show that the well-known Jacobian conjecture without any additional conditions is equivalent to what we call the vanishing conjecture: for any homogeneous polynomial $P(z)$ of degree $d=4$, if $\Delta^{m} P^{m}(z)=0$ for all $m \geq 1$, then $\Delta^{m} P^{m+1}(z)=0$ when $m>>0$, or equivalently, $\Delta^{m} P^{m+1}(z)=0$ when $m>\frac{3}{2}\left(3^{n-2}-1\right)$. It is also shown in this paper that the condition $\Delta^{m} P^{m}(z)=0(m \geq 1)$ above is equivalent to the condition that $P(z)$ is Hessian nilpotent, i.e. the Hessian matrix Hes $P(z)=\left(\frac{\partial^{2} P}{\partial z_{i} \partial z_{j}}\right)$ is nilpotent. The goal is achieved by using the recent breakthrough work of M. de Bondt, A. van den Essen and various results obtained in this paper on Hessian nilpotent polynomials. Some further results on Hessian nilpotent polynomials and the vanishing conjecture above are also derived.
\end{abstract}

\section{INTRODUCTION}

Let $z=\left(z_{1}, z_{2}, \cdots, z_{n}\right)$ and let $F(z)=z-H(z)$ be a formal map from $\mathbb{C}^{n}$ to $\mathbb{C}^{n}$ with $o(H(z)) \geq 2$ and $G(z)$ the formal inverse map of $F(z)$. The wellknown Jacobian conjecture first proposed by Keller [Ke in 1939 claims that if $F(z)$ is a polynomial map with the Jacobian $j(F)(z)=1$, the inverse map $G(z)$ must also be a polynomial map. Despite intense study from mathematicians in more than half a century, the conjecture is still wide open even for the case $n=2$. In 1998, S. Smale [S] included the Jacobian conjecture in his list of 18 important mathematical problems for the 21st century. For more history and known results on the Jacobian conjecture, see [BCW], E] and the references therein. Recently, M. de Bondt and A. van den Essen [BE1] (see also G. Meng [M]) have made a breakthrough on the Jacobian conjecture. They reduced the Jacobian conjecture to polynomial maps $F(z)=z-H(z)$ with $H(z)=\nabla P(z)=\left(\frac{\partial P}{\partial z_{1}}, \frac{\partial P}{\partial z_{2}}, \cdots, \frac{\partial P}{\partial z_{n}}\right)$ for some polynomials $P(z) \in \mathbb{C}[z]$. In this paper, we will refer to this reduction as the gradient reduction and the condition $H(z)=\nabla P(z)$ for some $P(z) \in \mathbb{C}[[z]]$ as the gradient condition. Note that, by the Poincaré lemma, a formal map $F(z)=$ $z-H(z)$ with $o(H(z)) \geq 2$ satisfies the gradient condition if and only if its Jacobian matrix $J F(z)$ is symmetric. Following the terminology in BE1, we also call the formal maps satisfying the gradient condition symmetric formal maps.

Received by the editors October 15, 2004 and, in revised form, October 26, 2004

2000 Mathematics Subject Classification. Primary 33C55, 39B32, 14R15, 31B05.

Key words and phrases. Hessian nilpotent polynomials, deformed inversion pairs, the heat equation, harmonic polynomials, the Jacobian conjecture.

(C)2006 American Mathematical Society Reverts to public domain 28 years from publication 
For further discussion, let us fix the following notions. A power series $P(z) \in$ $\mathbb{C}[[z]]$ is said to be HN (Hessian nilpotent) if its Hessian matrix Hes $P(z)=\left(\frac{\partial^{2} P}{\partial z_{i} \partial z_{j}}\right)$ is nilpotent. Let $t$ be a formal parameter which commutes with $z$. The deformed inversion pair $Q_{t}(z)$ of any $P(z) \in \mathbb{C}[[z]]$ with $o(P(z)) \geq 2$ is the unique power series $Q_{t}(z) \in \mathbb{C}[[z, t]]$ with $o\left(Q_{t}(z)\right) \geq 2$ such that the formal map $G(z)=z+t \nabla Q_{t}(z)$ is the inverse map of $F(z)=z-t \nabla P(z)$. Recently, G. Meng [M] and D. Wright Wr1] have derived a tree expansion formula for the inverse map $G(z)$ of $F(z)=F_{t=1}(z)$. In [Z2], by studying the PDE satisfied by the deformed inversion pairs $Q_{t}(z)$, the author has derived a recurrent formula and a binary rooted tree expansion formula for the deformed inversion pairs $Q_{t}(z)$. Furthermore, some close relationships among the deformed inversion pairs, the Legendre transform, the inviscid Burgers' equations and the Jacobian conjecture are also clarified in [Z2. For some other recent results on symmetric polynomial or formal maps, see [BE1]-BE5], [EW], [M], Wr1], Wr2 and [Z2].

In this paper, we will use some general results in Z2 to study HNS (Hessian nilpotent power series) $P(z)$ and their deformed inversion pairs $Q_{t}(z)$. Furthermore, by using the gradient reduction in BE1 and various results derived in this paper on HN polynomials, we will show that the Jacobian conjecture is equivalent to what we call vanishing conjectures of homogeneous HN polynomials. (See the discussion below.) We first derive the PDE's satisfied by $Q_{t}(z), \Delta^{k} Q_{t}^{m}(k, m \geq 1)$ and $\exp \left(s Q_{t}(z)\right)\left(s \in \mathbb{C}^{\times}\right)$, where $\Delta=\sum_{i=1}^{n} \frac{\partial^{2}}{\partial z_{i}^{2}}$ is the Laplace operator. In particular, we show in Theorem 3.2 that $\exp \left(s Q_{t}(z)\right)\left(s \in \mathbb{C}^{\times}\right)$is the unique power series solution of the Cauchy problem of the heat equation with the initial condition $\left.\exp \left(s Q_{t}(z)\right)\right|_{t=0}=\exp (s P(z))$. We then derive a uniform formula (see Theorem 3.4) for the powers $Q_{t}^{k}(z)(k \geq 1)$ of the deformed inversion pairs $Q_{t}(z)$ of HNS $P(z)$. We also prove a general theorem, Theorem 4.1, on a relationship between $\left\{\operatorname{Tr}_{\operatorname{Hes}^{m}}(P(z)) \mid m \geq 1\right\}$ and $\left\{\Delta^{m} P^{m}(z) \mid m \geq 1\right\}$ for any power series $P(z)$. From this theorem, we show in Theorem 4.3 that, for any formal power series $P(z)$, it is $\mathrm{HN}$ if and only if $\Delta^{m} P^{m}(z)=0$ for any $m \geq 1$, or equivalently, $\Delta^{m} P^{m}(z)=0$ for any $1 \leq m \leq n$. Finally, we prove some identities, vanishing properties and isotropic properties of $\left\{\Delta^{k} P^{m}(z) \mid m, k \geq 0\right\}$ for HNS or HNP's (Hessian nilpotent polynomials) $P(z)$. Some close relationships of the deformed pairs $Q_{t}(z)$ of HNS or HNP's $P(z)$ with the heat equation and the Jacobian conjecture are also clarified. In particular, we show that the Jacobian conjecture without any additional conditions is equivalent to the following vanishing conjectures: for any $\operatorname{HNP} P(z)$ of degree $d=4, \Delta^{m} P^{m+1}(z)=0$ for $m>>0$, or more precisely, for all $m>\frac{3}{2}\left(3^{n-2}-1\right)$.

One remark is that, due to the identity $\operatorname{Tr} \operatorname{Hes}(P)=\Delta P$, any HNS $P(z)$ is automatically harmonic, i.e. $\Delta P(z)=0$. Note that harmonic polynomials (see ABR, $[\mathrm{H}]$ and $[\mathrm{T}$ ) are among the most classical objects in mathematics and have been very well studied. The classical study on harmonic polynomials started from Legendre, Laplace, Jacobi in the late eighteenth century. The modern generalizations of harmonic polynomials, namely, spherical functions, were first studied by Cartan and Weyl in the 1930's and later by Gelfand, Harish-Chandra, etc. It is quite surprising to see that, first, HNP's as a family of very special harmonic polynomials are closely related with the notorious Jacobian conjecture. Second, it seems that HNP's have been overlooked and have not been studied until the recent work of M. de Bondt, A. van den Essen [BE1] and G. Meng [M]. Besides the connections with the Jacobian conjecture discussed above, another interesting aspect of HNP's 
is their connection with the classical inviscid Burgers' equation in diffusion theory and also the heat equation. Actually, the vanishing conjecture above is also equivalent to saying that the power series solutions of certain Cauchy problems of the inviscid Burgers' equation and the heat equation must be polynomials and the exponentials of polynomials, respectively. (See discussion in Section 4 in [Z2] and Conjecture 3.3 in this paper.) Considering the connections of HNP's with the classical objects described above, we believe that HNP's deserve much more attention from mathematicians.

Considering the length of this paper, we give the following detailed arrangement description. In Section 2, we first fix some notation and definitions that are needed throughout the rest of this paper. We then briefly recall certain results obtained in [Z2] and prove some preliminary results including the PDE (See Corollary 2.7) satisfied by $\Delta^{k} Q_{t}^{m}(k, m \geq 1)$ for the deformed inversion pairs $Q_{t}(z)$ of any power series $P(z)$. In Section 3, for any HNS $P(z)$ and its deformed inversion pair $Q_{t}(z)$, we derive the PDE's satisfied by $Q_{t}(z)$ and $\exp \left(s Q_{t}(z)\right)\left(s \in \mathbb{C}^{\times}\right)$, from which we derive with two different proofs a uniform formula (3.8) for $Q_{t}^{k}(z)(k \geq 1)$. In Section 4, we prove a general theorem, Theorem 4.1. on a relationship between $\left\{\operatorname{Tr} \operatorname{Hes}^{m}(P(z)) \mid m \geq 1\right\}$ and $\left\{\Delta^{m} P^{m}(z) \mid m \geq 1\right\}$ for the universal formal power series $P(z)$ with $o(P(z)) \geq 2$. From this theorem, we deduce a criterion in Theorem 4.3 for the Hessian nilpotency of a formal power series $P(z)$ in terms of certain vanishing properties of $\left\{\Delta^{m} P^{m}(z) \mid m \geq 1\right\}$.

In Section 5.1 by using a fundamental theorem of harmonic polynomials (see Theorem 5.2), we derive a criterion in Proposition 5.3 for Hessian nilpotency of homogeneous harmonic polynomials. In Section 5.2, we give constructions for some HNP's and HNS. In Section 6, by using some of the main results in the previous sections, we prove more properties of HNS or HNP's $P(z)$. We prove in Proposition 6.1 an identity and in Theorem 6.2 an equivalence of certain vanishing properties of $\left\{\Delta^{k} P^{m}(z) \mid k, m \geq 1\right\}$ for $\operatorname{HNS} P(z) \in \mathbb{C}[[z]]$. In Theorem 6.3, we show some isotropic properties for homogeneous HNP's. In Section 7 we discuss some applications to the Jacobian conjecture. We formulate the vanishing conjecture, Conjecture 7.1, for (not necessary homogeneous) HNP's and the homogeneous vanishing conjecture, Conjecture 7.3, for homogeneous HNP's. We show in Proposition 7.4 that both conjectures above are equivalent to the Jacobian conjecture.

Finally, some remarks on this paper are as follows. First, for convenience, we will fix $\mathbb{C}$ as our base field. But, all results, formulas as well as their proofs (except the first proof of Theorem 3.4) obtained in this paper hold or work equally well if one replaces $\mathbb{C}$ by any $\mathbb{Q}$-algebra. Second, we will not restrict our study to just HNP's. Instead, we will formulate and prove results for $\mathrm{HN}$ formal power series whenever they hold in this general setting. Third, for any HNP's or locally convergent HNS $P(z)$, all formal power series involved in this paper are locally convergent. This can be seen either from the fact that any local analytic map with non-zero Jacobian at the origin has a locally convergent inverse, or from the well-known CauchyKowaleskaya theorem (see $\underline{\mathrm{R}}$, for example).

\section{DEFormed INVERSION PAIRS OF FORMAL POWER SERIES}

In this section, we first fix some notation and definitions that are needed in this paper. We then briefly recall certain results obtained in [Z2] and prove some preliminary results. 
2.1. Notation and conventions. Once and for all, we fix the following notation and conventions:

(1) We fix $n \geq 1$ and set $z=\left(z_{1}, z_{2}, \cdots, z_{n}\right)$. For any $\mathbb{Q}$-algebra $k$, we denote by $k[z]$ (resp. $k[[z]])$ the polynomial algebra (resp. formal power series algebra) over $k$ in $z_{i}(1 \leq i \leq n)$.

(2) For any $\mathbb{Q}$-algebra $k$, by a formal map $F(z)$ from $k^{n}$ to $k^{n}$, we simply mean $F(z)=\left(F_{1}(z), F_{2}(z), \cdots, F_{n}(z)\right)$ with $F_{i}(z) \in k[[z]](1 \leq i \leq n)$. We denote by $J(F)$ and $j(F)$ the Jacobian matrix and the Jacobian of $F(z)$, respectively.

(3) We denote by $\Delta$ the Laplace operator $\sum_{i=1}^{n} \frac{\partial^{2}}{\partial z_{i}^{2}}$. Note that a polynomial or formal power series $P(z)$ is said to be harmonic if $\Delta P=0$.

(4) For any $k \geq 1$ and $U(z)=\left(U_{1}(z), U_{2}(z), \cdots, U_{k}(z)\right) \in \mathbb{C}[[z]]^{\times k}$, we set

$$
o(U(z))=\min _{1 \leq i \leq k} o\left(U_{i}(z)\right)
$$

and, when $U(z) \in \mathbb{C}[z]^{\times k}$,

$$
\operatorname{deg} U(z)=\max _{1 \leq i \leq k} \operatorname{deg} U_{i}(z)
$$

For any $U_{t}(z) \in \mathbb{C}[t][[z]]^{\times k}$ or $\mathbb{C}[[z, t]]^{\times k}(k \geq 1)$ for some formal parameter $t$, the notation $o\left(U_{t}(z)\right)$ and $\operatorname{deg} U_{t}(z)$ always stand for the order and the degree of $U_{t}(z)$ with respect to $z$, respectively.

(5) For any $P(z) \in \mathbb{C}[[z]]$, we denote by $\nabla P(z)$ the gradient of $P(z)$, i.e. $\nabla P=$ $\left(\frac{\partial P}{\partial z_{1}}, \frac{\partial P}{\partial z_{2}}, \cdots, \frac{\partial P}{\partial z_{n}}\right)$. We denote by Hes $(P)(z)$ the Hessian matrix of $P(z)$, i.e. $\operatorname{Hes}(P)(z)=\left(\frac{\partial^{2} P(z)}{\partial z_{i} \partial z_{j}}\right)$.

(6) All $n$-vectors in this paper are assumed to be column vectors unless stated otherwise. For any vector or matrix $U$, we denote by $U^{t}$ its transpose. The standard $\mathbb{C}$-bilinear form of $n$-vectors is denoted by $\langle\cdot, \cdot\rangle$.

The following lemma will be very useful in our later arguments.

Lemma 2.1. For any $P(z) \in \mathbb{C}[[z]]$ and $m \geq 1$, we have

$$
\Delta P^{m+1}(z)=(m+1) P^{m} \Delta P+m(m+1) P^{m-1}\langle\nabla P, \nabla P\rangle
$$

or

$$
P^{m-1}\langle\nabla P, \nabla P\rangle=\frac{1}{m(m+1)}\left(\Delta P^{m+1}-(m+1) P^{m} \Delta P\right) .
$$

Furthermore, when $P(z)$ is harmonic, we have

$$
P^{m-1} \Delta P^{2}=\frac{2}{m(m+1)} \Delta P^{m+1}
$$


Proof. Consider

$$
\begin{aligned}
\Delta P^{m+1} & =\sum_{i=1}^{n} \frac{\partial}{\partial z_{i}} \frac{\partial}{\partial z_{i}} P^{m+1} \\
& =(m+1) \sum_{i=1}^{n} \frac{\partial}{\partial z_{i}}\left(P^{m} \frac{\partial P}{\partial z_{i}}\right) \\
& =(m+1) \sum_{i=1}^{n} P^{m} \frac{\partial^{2} P}{\partial z_{i}^{2}}+m(m+1) \sum_{i=1}^{n} P^{m-1} \frac{\partial P}{\partial z_{i}} \frac{\partial P}{\partial z_{i}} \\
& =(m+1) P^{m} \Delta P+m(m+1)\langle\nabla P, \nabla P\rangle P^{m-1} .
\end{aligned}
$$

Hence, we get (2.1) and (2.2).

Now suppose that $P(z)$ is harmonic, i.e. $\Delta P=0$. By (2.1) with $m=1$, we have $\Delta P^{2}=2\langle\nabla P, \nabla P\rangle$ or $\langle\nabla P, \nabla P\rangle=\frac{1}{2} \Delta P^{2}$. It is easy to see that, in this case, (2.3) follows directly from (2.2).

2.2. Deformed inversion pairs of formal power series. For any $P(z) \in \mathbb{C}[[z]]$ with $o(P(z)) \geq 2$, we set $F(z)=z-\nabla P(z)$. It is shown in $\S 1.1$ in $[\mathrm{M}$ ] (see also Lemma 3.1 in [Z2]) that there is a unique $Q(z) \in \mathbb{C}[[z]]$ with $o(Q(z)) \geq 2$ such that the formal inverse of $F(z)$ is given by $G(z)=z+\nabla Q(z)$. We call $Q(z)$ the inversion pair of $P(z)$. Furthermore, following the arguments in [Z1] and [Z2], we also consider the deformation $F_{t}(z)=z-t \nabla P(z)$ of $F(z)$, where $t$ is a formal parameter which commutes with variables $z_{i}(1 \leq i \leq n)$. By Lemma 3.1 in [Z2], we know that there exists a unique $Q_{t}(z) \in \mathbb{C}[[z, t]]$ with $o\left(Q_{t}(z)\right) \geq 2$ such that the formal inverse $G_{t}(z)$ of $F_{t}(z)$ is given by $G_{t}(z)=z+t \nabla Q_{t}(z)$. Note that, when $o(P(z)) \geq 3$, we actually have $Q_{t}(z) \in \mathbb{C}[t][[z]]$ and $Q_{t=1}(z)=Q(z)$. In general, $t Q_{t}(z)$ is nothing but the inversion pair of $t P(z)$ over the $\mathbb{Q}$-algebra $\mathbb{C}[[t]]$. Another way to look at the inversion pair is as follows. Set $U(z)=\frac{1}{2} \sum_{i=1}^{n} z_{i}^{2}-P(z)$ and $V(z)=\frac{1}{2} \sum_{i=1}^{n} z_{i}^{2}+Q(z)$ then $V(z)$ is exactly the Legendre transform (see $\mathrm{Ar}$, [M] and [Z2]) of the formal power series $U(z)$.

Definition 2.2. For any $P(z) \in \mathbb{C}[[z]]$ with $o(P(z)) \geq 2, Q_{t}(z) \in \mathbb{C}[[z, t]]$ defined above is called the deformed inversion pair of $P(z)$.

Another important definition is the following.

Definition 2.3. For any $P(z) \in \mathbb{C}[[z]]$, we say $P(z)$ is $\mathrm{HN}$ (Hessian nilpotent) if its Hessian matrix Hes $(P)=\left(\frac{\partial^{2} P}{\partial z_{i} \partial z_{j}}\right)$ is nilpotent.

Remark 2.4. Note that $\operatorname{Tr} \operatorname{Hes}(P)=\Delta P$ for any $P(z) \in \mathbb{C}[[z]]$. Hence any HN formal power series is harmonic. But the converse is not true. For some examples of HNP's and HNS, see Subsection 5.2,

Throughout the rest of this paper, for any formal power series $P(z) \in \mathbb{C}[[z]]$, we will fix the notation $F(z), F_{t}(z), G(z), G_{t}(z), Q(z)$ and $Q_{t}(z)$ defined above unless stated otherwise. For short, we will use HNS and HNP for "HN power series" and "HN polynomial", respectively. Furthermore, we also define a sequence of formal power series $\left\{Q_{[m]}(z) \mid m \geq 1\right\}$ by writing

$$
Q_{t}(z)=\sum_{m=1}^{\infty} t^{m-1} Q_{[m]}(z) .
$$


Lemma 2.5. For any formal power series $P(z)$, we have

(a)

$$
\left(\Delta Q_{t}\right)\left(F_{t}\right)=\sum_{k=1}^{\infty} t^{k-1} \operatorname{Tr} \operatorname{Hes}^{k}(P) .
$$

(b) $P(z)$ is $H N$ if and only if $Q_{t}(z)$ is harmonic as a formal power series in $z$, and if and only if $Q_{t}(z)$ is $H N$ as a formal power series in $z$.

Proof. (a) Set $N_{t}(z)=\nabla Q_{t}(z)$. It is easy to check that

$$
\begin{array}{r}
J N_{t}(z)=\operatorname{Hes} Q_{t}(z), \\
\operatorname{Tr} \operatorname{Hes}\left(Q_{t}\right)=\Delta Q_{t} .
\end{array}
$$

Then (2.5) follows directly from the equations above and (2.4) in [Z2].

(b) follows directly by applying Lemma 2.2 in [Z2] to the formal map $F(z)=$ $z-H(z)$ with $H(z)=\nabla P(z)$.

The following theorem which was first proved in the unpublished preprint [Z1] and later [Z2] (see Theorem 3.6 and Proposition 3.7 in [Z2]) will play a fundamental role in this paper.

Theorem 2.6 $([\mathrm{Z} 2])$. For any $Q_{t}(z) \in \mathbb{C}[[z, t]]$ with $o\left(Q_{t}(z)\right) \geq 2$ and $P(z) \in \mathbb{C}[[z]]$ with $o(P(z)) \geq 2$, the following statements are equivalent:

(1) $Q_{t}(z)$ is the deformed inversion pair of $P(z)$.

(2) $Q_{t}(z)$ is the unique power series solution of the following Cauchy problem of PDE's:

$$
\left\{\begin{array}{l}
\frac{\partial Q_{t}(z)}{\partial t}=\frac{1}{2}\left\langle\nabla Q_{t}, \nabla Q_{t}\right\rangle \\
Q_{t=0}(z)=P(z)
\end{array}\right.
$$

Furthermore, we have the following recurrent formula:

$$
\begin{aligned}
Q_{[1]}(z) & =P(z), \\
Q_{[m]}(z) & =\frac{1}{2(m-1)} \sum_{\substack{k, l \geq 1 \\
k+l=m}}\left\langle\nabla Q_{[k]}(z), \nabla Q_{[l]}(z)\right\rangle
\end{aligned}
$$

for any $m \geq 2$.

Corollary 2.7. For any $k \geq 0$ and $m \geq 1$, we have

$$
\frac{\partial}{\partial t} \Delta^{k} Q_{t}^{m}(z)=\frac{1}{2(m+1)} \Delta^{k+1} Q_{t}^{m+1}-\frac{1}{2} \Delta^{k}\left(Q_{t}^{m} \Delta Q_{t}\right) .
$$

Proof. Since $\frac{\partial}{\partial t}$ and $\Delta^{k}(k \geq 0)$ commute, by applying $\Delta^{k}(k \geq 1)$ to (2.11) with $k=0$, we get (2.11) for any $k \geq 1$. Therefore we may assume $k=0$.

Consider

$$
\frac{\partial}{\partial t} Q_{t}^{m}(z)=m Q_{t}^{m-1} \frac{\partial Q_{t}}{\partial t}
$$

Applying (2.8) in Theorem 2.6.

$$
=\frac{m}{2} Q_{t}^{m-1}\left\langle\nabla Q_{t}, \nabla Q_{t}\right\rangle .
$$


Applying (2.2) in Lemma 2.1 to $Q_{t}(z)$ :

$$
\begin{aligned}
& =\frac{m}{2} \frac{1}{m(m+1)}\left(\Delta Q_{t}^{m+1}-(m+1) Q_{t}^{m} \Delta Q_{t}\right) \\
& =\frac{1}{2(m+1)} \Delta Q_{t}^{m+1}-\frac{1}{2} Q_{t}^{m} \Delta Q_{t} .
\end{aligned}
$$

\section{DEFORMED INVERSION PAIRS OF HN POWER SERIES}

In this section, we study deformed inversion pairs $Q_{t}(z)$ of HNS (Hessian Nilpotent Formal Power Series) $P(z) \in \mathbb{C}[[z]]$. We first derive the PDE's satisfied by $Q_{t}(z)$ and $\exp \left(s Q_{t}(z)\right)\left(s \in \mathbb{C}^{\times}\right)$. We then discuss some relationships among deformed inversion pairs, the Heat equation and the Jacobian conjecture. Note that similar relationships among deformed inversion pairs of formal power series (not necessarily HN), the inviscid Burgers' equations and the Jacobian conjecture have been discussed in [Z2. Finally, we derive with two different proofs a uniform nonrecurrent formula (see (3.8) ) for $Q_{t}^{k}(z)(k \geq 1)$.

Theorem 3.1. For any $Q_{t}(z) \in \mathbb{C}[[z, t]]$ with o $\left(Q_{t}(z)\right) \geq 2$ and $H N S P(z) \in \mathbb{C}[[z]]$ with $o(P(z)) \geq 2$, the following statements are equivalent:

(1) $Q_{t}(z)$ is the deformed inversion pair of $P(z)$.

(2) $Q_{t}(z)$ is the unique power series solution of the following Cauchy problem of PDE:

$$
\begin{cases}\frac{\partial Q_{t}(z)}{\partial t} & =\frac{1}{4} \Delta Q_{t}^{2} \\ Q_{t=0}(z) & =P(z)\end{cases}
$$

Furthermore, we have the following recurrent formula:

$$
\begin{aligned}
Q_{[1]}(z) & =P(z), \\
Q_{[m]}(z) & =\frac{1}{4(m-1)} \Delta \sum_{\substack{k, l \geq 1 \\
k+l=m}} Q_{[k]}(z) Q_{[l]}(z)
\end{aligned}
$$

for any $m \geq 2$.

Proof. First, by Lemma 2.5(b), we have $\Delta Q_{t}(z)=0$. Therefore, for any $m \geq 1$, $Q_{[m]}(z)$ is harmonic. Second, for any harmonic formal power series $U(z), \mathrm{V}(\mathrm{z}) \in$ $\mathbb{C}[[z]]$, it is easy to check that we have

$$
\Delta(U V)=2\langle\nabla U, \nabla V\rangle .
$$

By using the facts above, it is easy to see that the implication $(1) \Rightarrow(2)$ and also the recurrent formulas (3.2), (3.3) follow directly from Theorem 2.6.

To see $(2) \Rightarrow(1)$, we denote by $\widetilde{Q}_{t}(z)$ the deformed inversion pair of $P(z)$. By the fact proved above, we know that $\widetilde{Q}_{t}(z)$ also satisfies (3.1). Since the power series solution of the Cauchy problem (3.1) is unique, which is given recursively by (3.2) and (3.3), we have $\widetilde{Q}_{t}(z)=Q_{t}(z)$. Therefore $(2) \Rightarrow(1)$ also holds.

A relation of deformed inversion pairs $Q_{t}(z)$ of HNS $P(z)$ with the heat equation is given by the following theorem. 
Theorem 3.2. Let $P(z) \in \mathbb{C}[[z]]$ be $H N$ with $o(P(z)) \geq 2$ and $Q_{t}(z)$ its deformed inversion pair. For any non-zero $s \in \mathbb{C}$, set

$$
U_{t, s}(z)=\exp \left(s Q_{t}(z)\right)=\sum_{k=0}^{\infty} \frac{s^{k} Q_{t}^{k}(z)}{k !} .
$$

Then, $U_{t, s}(z)$ is the unique formal power series solution of the following Cauchy problem of the Heat equation:

$$
\begin{cases}\frac{\partial U_{t, s}}{\partial t}(z) & =\frac{1}{2 s} \Delta U_{t, s}(z) \\ U_{t=0, s}(z) & =\exp (s P(z)) .\end{cases}
$$

Proof. The uniqueness can be proved by viewing $U_{t, s}(z)$ as a power series in $t$ with coefficients in $\mathbb{C}[[z]]$ and showing that the coefficients of $t^{k}(k \geq 1)$ are recurrently determined by the coefficient of $t^{0}$ which is $U_{t=0, s}(z)=\exp (s P(z))$. We skip the details here. For a similar argument, see the proof of Proposition 2.5 in [2]. Note that, when $P(z)$ is locally convergent, $Q_{t}(z)$ and $\exp \left(s Q_{t}(z)\right)\left(s \in \mathbb{C}^{\times}\right)$are locally convergent. Then the uniqueness in this case also follows from the CauchyKowaleskaya theorem (see $[\mathbf{R})$ in PDE.

Now we show that $U_{t, s}(z)$ satisfies (3.5). First note that the initial condition in (3.5) follows immediately from the one in (2.8). Second, by Lemma 2.5 (b), we have $\Delta Q_{t}(z)=0$.

Consider

$$
\begin{aligned}
\frac{\partial U_{t, s}}{\partial t} & =\frac{\partial \exp \left(s Q_{t}\right)}{\partial t} \\
& =s \exp \left(s Q_{t}\right) \frac{\partial Q_{t}}{\partial t}
\end{aligned}
$$

Applying (2.8):

$$
=\frac{s}{2} U_{t, s}\left\langle\nabla Q_{t}, \nabla Q_{t}\right\rangle .
$$

On the other hand, we have

$$
\begin{aligned}
\Delta U_{t, s} & =\sum_{i=1}^{n} \frac{\partial}{\partial z_{i}} \frac{\partial}{\partial z_{i}} e^{s Q_{t}} \\
& =s \sum_{i=1}^{n} \frac{\partial}{\partial z_{i}}\left(\frac{\partial Q_{t}}{\partial z_{i}} e^{s Q_{t}}\right) .
\end{aligned}
$$

Using the fact that $\Delta Q_{t}=0$ :

$$
\begin{aligned}
& =s^{2} \sum_{i=1}^{n} \frac{\partial Q_{t}}{\partial z_{i}} \frac{\partial Q_{t}}{\partial z_{i}} e^{s Q_{t}} \\
& =s^{2} U_{s, t}\left\langle\nabla Q_{t}, \nabla Q_{t}\right\rangle .
\end{aligned}
$$

By combining (3.6) and (3.7), we see that $U_{t, s}(z)$ does satisfy the PDE in (3.5).

By combining the gradient reduction in [BE1], $\mathrm{M}]$ and the homogeneous reduction in $[\mathrm{BCW},[\mathrm{Y}]$ on the Jacobian conjecture, we see that the Jacobian conjecture 
can be reduced to polynomial maps $F(z)=z-\nabla P(z)$ with $P(z) \in \mathbb{C}[z]$ homogeneous of degree $d=4$. By Theorems 3.1 and 3.2, it is easy to see that the Jacobian conjecture is equivalent to the following conjecture.

Conjecture 3.3. For any homogeneous $H N P P(z)$ of degree $d \geq 2$, the unique solutions of the Cauchy problems (3.5) and (2.8) must be a polynomial in $(z, t)$ and the exponential of a polynomial $(z, t)$, respectively.

Since it has been proved by Wang Wa that the Jacobian conjecture holds for polynomial maps $F(z)$ of $\operatorname{deg} F(z) \leq 2$, Conjecture 3.3 is true for $d \leq 3$. For more discussion on relationships of HNP's and the Jacobian conjecture, see Section 7

Next we give two different proofs for the following uniform formula for the powers $Q_{t}^{k}(z)(k \geq 1)$ of the deformed inversion pairs $Q_{t}(z)$ of HNS $P(z)$.

Theorem 3.4. Suppose $P(z) \in \mathbb{C}[[z]]$ with $o(P(z)) \geq 2$ is $H N$. Then, for any $k \geq 1$, we have

$$
Q_{t}^{k}(z)=k ! \sum_{m=0}^{\infty} \frac{t^{m}}{2^{m} m !(m+k) !} \Delta^{m} P^{m+k}(z) .
$$

In particular, for any $m \geq 1$,

$$
Q_{[m]}(z)=\frac{1}{2^{m-1} m !(m-1) !} \Delta^{m-1} P^{m}(z) .
$$

First proof. First, note that (3.9) follows directly from (3.8) with $k=1$ and the definition (2.4) of $Q_{[m]}(z)(m \geq 1)$. To prove (3.8), we consider the formal power series

$$
\exp \left(\frac{t}{2 s} \Delta\right) \exp (s P)=\sum_{k=0}^{\infty} \frac{t^{k}}{(2 s)^{k} k !} \Delta^{k} \exp (s P) .
$$

It is easy to check that the series above is also a formal power series solution of the Cauchy problem (3.5). Hence, by Theorem 3.2 and the uniqueness of the power series solution of (3.5), we have

$$
\exp \left(s Q_{t}\right)=\exp \left(\frac{t}{2 s} \Delta\right) \exp (s P) .
$$

By comparing the coefficients of $s^{k}(k \geq 1)$ to both sides of the equation above, we get (3.8).

The above proof for Theorem 3.4 is shorter but less intriguing than the second proof below, which begins with the following lemma.

Lemma 3.5. Let $P(z) \in \mathbb{C}[[z]]$ with $o(P(z)) \geq 2$ be $H N$ and $Q_{t}(z)$ the deformed inversion pair of $P(z)$. Then, for any $k, l \geq 1$, we have

$$
\frac{\partial^{l} Q_{t}^{k}}{\partial t^{l}}=\frac{\Delta^{l} Q_{t}^{k+l}}{2^{l}(k+1)(k+2) \cdots(k+l)}
$$

Proof. We fix $k \geq 1$ and use the mathematical induction on $l \geq 1$. First, by Lemma 2.5(b), we have $\Delta Q_{t}(z)=0$. Then (3.12) for $l=1$ follows directly from (2.11). 
Now we assume that (3.12) holds for $l=l_{0} \geq 1$ and consider the case $l=l_{0}+1$ :

$$
\begin{aligned}
\frac{\partial^{l} Q_{t}^{k}}{\partial t^{l}} & =\frac{\partial}{\partial t}\left(\frac{\partial^{l_{0}} Q_{t}^{k}}{\partial t^{l_{0}}}\right) \\
& =\frac{1}{2^{l_{0}}(k+1)(k+2) \cdots\left(k+l_{0}\right)} \frac{\partial}{\partial t} \Delta^{l_{0}} Q_{t}^{k+l_{0}} \\
& =\frac{k+l_{0}}{2^{l_{0}}(k+1)(k+2) \cdots\left(k+l_{0}\right)} \Delta^{l_{0}}\left(Q_{t}^{k+l_{0}-1} \frac{\partial Q_{t}}{\partial t}\right) .
\end{aligned}
$$

Applying the PDE in (3.1):

$$
=\frac{1}{2^{l_{0}}(k+1)(k+2) \cdots\left(k+l_{0}-1\right)} \frac{1}{4} \Delta^{l_{0}}\left(Q_{t}^{k+l_{0}-1} \Delta Q_{t}^{2}\right) .
$$

Applying (2.3) to $Q_{t}^{k+l_{0}-1} \Delta Q_{t}^{2}$ :

$$
\begin{aligned}
& =\frac{1}{2^{l_{0}+2}(k+1) \cdots\left(k+l_{0}-1\right)} \frac{2}{\left(k+l_{0}\right)\left(k+l_{0}+1\right)} \Delta^{l_{0}+1} Q_{t}^{k+l_{0}+1} \\
& =\frac{1}{2^{l_{0}+1}(k+1)(k+2) \cdots\left(k+l_{0}+1\right)} \Delta^{l_{0}+1} Q_{t}^{k+l_{0}+1} \\
& =\frac{1}{2^{l}(k+1)(k+2) \cdots(k+l)} \Delta^{l} Q_{t}^{k+l} .
\end{aligned}
$$

Hence, (3.12) holds for $l=l_{0}+1$.

Second proof of Theorem 3.4. First, by the initial condition in (2.8), we have

$$
\left.\Delta^{l} Q_{t}^{k+l}(z)\right|_{t=0}=\Delta^{l} P^{k+l}(z)
$$

for any $k \geq 1$ and $l \geq 0$.

Second, by setting $t=0$ in (3.12) and applying the equation above, we see that the coefficient of $t^{l}$ of $Q_{t}^{k}(z)$ is equal to

$$
\frac{1}{l !} \frac{\Delta^{l} P^{k+l}}{2^{l}(k+1)(k+2) \cdots(k+l)}=\frac{k !}{2^{l} l !(k+l) !} \Delta^{l} P^{k+l} .
$$

Hence (3.8) holds.

By comparing the coefficients of $s^{k}(k \leq 0)$ on both sides of (3.10), we see that $\Delta^{k} P^{m}=0$ for any $k \geq m$, which is equivalent to saying that $\Delta^{m} P^{m}=0$ for any $m \geq 1$. Note that the latter statement also follows from (3.8) with $k=1$ and the fact that $Q_{t}(z)$ is harmonic.

Corollary 3.6. For any $H N S P(z) \in \mathbb{C}[[z]]$ with $o(P(z)) \geq 2$, we have $\Delta^{m} P^{m}=0$ for any $m \geq 1$.

Later, we will show in Theorem 4.3 that the converse of the corollary above is also true.

Note that, by setting $s=1$ in (3.11), we have the following formula:

$$
\exp \left(Q_{t}\right)=\exp \left(\frac{t}{2} \Delta\right) \exp (P)
$$

Actually, a more delicate formula (see (3.20) below) can be derived as follows. 
Set

$$
\begin{aligned}
& \widetilde{Q}_{t}(z)=Q_{t}(z)-P(z) \\
& \widetilde{U}_{t}(z)=\exp \left(\widetilde{Q}_{t}(z)\right) \\
& \Lambda_{P}(z)=\sum_{i=1}^{n} \frac{\partial P(z)}{\partial z_{i}} \frac{\partial}{\partial z_{i}}
\end{aligned}
$$

Lemma 3.7. For any $\operatorname{HNS} P(z) \in \mathbb{C}[[z]]$ with $o(P(z)) \geq 2$, let $\widetilde{Q}_{t}(z), \widetilde{U}_{t}(z)$ and $\Lambda_{P}$ be as above. Then we have

(a) $\widetilde{Q}_{t}(z)$ is the unique power series solution of the following Cauchy problem of $P D E$ 's:

$$
\begin{cases}\frac{\partial \widetilde{Q}_{t}(z)}{\partial t} & =\frac{1}{4} \Delta \widetilde{Q}_{t}^{2}(z)+\Lambda_{P} \widetilde{Q}_{t}(z)+\frac{1}{4} \Delta P^{2} \\ \widetilde{Q}_{t=0}(z) & =0\end{cases}
$$

(b) $\widetilde{U}_{t}(z)$ is the unique power series solution of the following Cauchy problem of $P D E$ 's:

$$
\begin{cases}\frac{\partial \widetilde{U}_{t}(z)}{\partial t} & =\left(\frac{1}{2} \Delta+\Lambda_{P}+\frac{1}{4} \Delta P^{2}\right) \widetilde{U}_{t}(z), \\ \widetilde{U}_{t=0}(z) & =1 .\end{cases}
$$

The proof of this lemma is straightforward and similar as in the proof of Theorem 3.2, so we omit it here. From (3.19), it is also easy to derive the following formula.

Proposition 3.8. For any $H N S P(z) \in \mathbb{C}[[z]]$ with $o(P(z)) \geq 2$, we have

$$
\exp \left(\widetilde{Q}_{t}\right)=\exp \left(t\left(\frac{1}{2} \Delta+\Lambda_{P}+\frac{1}{4} \Delta P^{2}\right)\right) \cdot 1 .
$$

Proof. We first set

$$
V_{t}(z)=\exp \left(t\left(\frac{1}{2} \Delta+\Lambda_{P}+\frac{1}{4} \Delta P^{2}\right)\right) \cdot 1 .
$$

Note that $V_{t=0}(z)=1$. Now we consider

$$
\begin{aligned}
\frac{\partial V_{t}}{\partial t} & =\left(\frac{1}{2} \Delta+\Lambda_{P}+\frac{1}{4} \Delta P^{2}\right) \exp \left(t\left(\frac{1}{2} \Delta+\Lambda_{P}+\frac{1}{4} \Delta P^{2}\right)\right) \cdot 1 \\
& =\left(\frac{1}{2} \Delta+\Lambda_{P}+\frac{1}{4} \Delta P^{2}\right) V_{t}(z) .
\end{aligned}
$$

Therefore $V_{t}(z)$ satisfies the PDE in (3.19). On the other hand, by Lemma 3.7(b) above, $\exp \left(\widetilde{Q}_{t}\right)$ also satisfies (3.19). Hence, (3.20) follows from the uniqueness of power series solutions of the Cauchy problem (3.19).

One interesting aspect of the formula above is as follows. It shows that the differential operator $\Lambda_{P}$ and the operator of the multiplication by $\Delta P^{2}$ also play important roles for deformed inversion pairs $Q_{t}(z)$. For example, from (3.20), it is easy to see that we have the following corollary.

Corollary 3.9. For any $P(z) \in \mathbb{C}[[z]]$ with $o(P(z)) \geq 2$ such that $\Delta P^{2}(z)=0$, we have $\widetilde{Q}_{t}(z)=0$, or in other words, $Q_{t}(z)=P(z)$. 


\section{A CRITERION fOR HeSSIAN NILPOTENCY}

Let $a=\left\{a_{I}\left|I \in \mathbb{N}^{n},\right| I \mid \geq 2\right\}$ be a set of variables that commute with each other. Let $P(z)=\sum_{I \in \mathbb{N}^{n}} a_{I} z^{I}$ be the universal formal power series in $z$ with $o(P(z)) \geq 2$. We will also view $P(z)$ as a formal power series in $z$ with coefficients in $\mathbb{C}[a]$, i.e. $P(z) \in \mathbb{C}[a][[z]]$.

For any $m \geq 1$, we set

$$
\begin{aligned}
& u_{m}(P)=\operatorname{Tr}_{\text {Hes }}^{m}(P), \\
& v_{m}(P)=\Delta^{m} P^{m} .
\end{aligned}
$$

In this section, we prove a general theorem, Theorem 4.1 about a relation between $\left\{u_{m}(P) \mid m \geq 1\right\}$ and $\left\{v_{m}(P) \mid m \geq 1\right\}$. Consequently, we get a criterion for Hessian nilpotency of formal power series $P(z) \in \mathbb{C}[[z]]$ in terms of certain vanishing properties of $\left\{\Delta^{m} P^{m}(z) \mid m \geq 1\right\}$.

Let $P(z) \in \mathbb{C}[a][[z]],\left\{u_{m}(P) \mid m \geq 1\right\}$ and $\left\{v_{m}(P) \mid m \geq 1\right\}$ be defined as above.

For any $k \geq 1$, we define $\mathcal{U}_{k}(P)$ (resp. $\left.\mathcal{V}_{k}(P)\right)$ to be the ideal in $\mathbb{C}[a][[z]]$ generated by $\left\{u_{m}(P) \mid 1 \leq m \leq k\right\}$ (resp. $\left.\left\{v_{m}(P) \mid 1 \leq m \leq k\right\}\right)$ and all their partial derivatives of any order. For convenience, we also set $\mathcal{U}_{0}(P)=\mathcal{V}_{0}(P)=0$.

The first main result of this subsection is the following theorem.

Theorem 4.1. For any $k \geq 1, \mathcal{U}_{k}(P)=\mathcal{V}_{k}(P)$ as ideals in $\mathbb{C}[a][[z]]$.

One immediate consequence of Theorem 4.1 is the following corollary.

For any $k \geq 1$, we define $\widetilde{\mathcal{U}}_{k}(P)$ (resp. $\left.\widetilde{\mathcal{V}}_{k}(P)\right)$ to be the ideal in $\mathbb{C}[a]$ generated by all coefficients of $\left\{u_{m}(P) \mid 1 \leq m \leq k\right\}$ (resp. $\left\{v_{m}(P) \mid 1 \leq m \leq k\right\}$ ).

Corollary 4.2.1 For any $k \geq 1, \widetilde{\mathcal{U}}_{k}(P)=\widetilde{\mathcal{V}}_{k}(P)$ as ideals in $\mathbb{C}[a]$.

From Theorem 4.1 or Corollary 4.2, it is easy to see that we have the following criteria for Hessian nilpotency of formal power series $P(z) \in \mathbb{C}[[z]]$.

Theorem 4.3. For any $P(z) \in \mathbb{C}[[z]]$ with $o(P(z)) \geq 2$, the following statements are equivalent:

(1) $P(z)$ is $H N$.

(2) $\Delta^{m} P^{m}=0$ for any $m \geq 1$.

(3) $\Delta^{m} P^{m}=0$ for any $1 \leq m \leq n$.

Proof. By Theorem 4.1 or Corollary 4.2, we have, for any fixed $k \geq 1, \Delta^{m} P^{m}=0$ $(1 \leq m \leq k)$ if and only if $\operatorname{Tr} \operatorname{Hes}^{m}(P)=0(1 \leq m \leq k)$. Then, the theorem follows directly from the following facts in linear algebra, namely, for any $n \times n$ matrix $A \in M_{n}(\mathbb{C}), A$ is nilpotent if and only if $\operatorname{Tr} A^{m}=0(m \geq 1)$, and if and only if $\operatorname{Tr} A^{m}=0(1 \leq m \leq n)$.

Besides the criteria in Theorem 4.3, we believe the following is also true.

Conjecture 4.4. Let $P(z) \in \mathbb{C}[[z]]$ with $o(P(z)) \geq 2$. If $\Delta^{m} P^{m}(z)=0$ for $m>>$, then $P(z)$ is $H N$.

In the rest of this subsection, we fix the universal formal power series $P(z)=$ $\sum_{I \in \mathbb{N}^{n}} a_{I} z^{I}$ with $o(P(z)) \geq 2$ and give a proof for Theorem 4.1. Note that all results

\footnotetext{
${ }^{1}$ Professor David Wright Wr3 recently informed the author that he has obtained a different proof for this result.
} 
proved in the previous sections also hold for formal power series over the $\mathbb{C}$-algebra $\mathbb{C}[a]$. In particular, they hold for our universal formal power series $P(z) \in \mathbb{C}[a][[z]]$.

We begin with the following two lemmas.

Lemma 4.5. Let $Q_{t}(z) \in \mathbb{C}[a][[z, t]]$ be the deformed inversion pair of $P(z)$. Then there exists a sequence $\left\{w_{k}(P)(z) \in \mathcal{U}_{k}(P) \mid k \geq 1\right\}$ such that

$$
\Delta Q_{t}(z)=\sum_{k=1}^{\infty} w_{k}(P)(z) t^{k-1}
$$

and, for any $k \geq 1$,

$$
w_{k}(P)(z) \equiv u_{k}(P)(z) \quad \bmod \left(\mathcal{U}_{k-1}(P)\right) .
$$

Proof. We set $N_{t}(z)=\nabla Q_{t}(z)$. First, by composing $G_{t}(z)=z+t N_{t}(z)$ from the right to (2.5) in Lemma 2.5, we have

$$
\Delta Q_{t}(z)=\sum_{i=1}^{\infty} u_{i}(P)\left(z+t N_{t}(z)\right) t^{i-1} .
$$

Now we write the Taylor expansion of $u_{i}(P)\left(z+t N_{t}(z)\right)(i \geq 1)$ at $z$ as

$$
u_{i}(P)\left(z+t N_{t}(z)\right)=u_{i}(P)(z)+\sum_{k=1}^{\infty} \sum_{\substack{\mathbf{s} \in \mathbb{N}^{n} \\|\mathbf{s}|=k}} \frac{1}{\mathbf{s} !} \frac{\partial^{\mathbf{s}} u_{i}(P)}{\partial z^{\mathbf{s}}}(z) N_{t}^{\mathbf{s}}(z) t^{k} .
$$

Next we want to write the RHS of (4.6) as a formal power series in $t$ with coefficients in $\mathbb{C}[[a, z]]$. This can be done by first doing so for $N_{t}^{\mathbf{s}}(z)\left(\mathbf{s} \in \mathbb{N}^{n}\right)$ in (4.6) and then properly re-arranging all the terms involved. Note that $u_{i}(P)(z)$ or $\frac{\partial^{\mathbf{s}} u_{i}(P)}{\partial z^{\mathbf{s}}}(z)\left(\mathbf{s} \in \mathbb{N}^{n}\right)$ do not depend on $t$ and are in the ideal $\mathcal{U}_{i}(P)$. Also note that all the terms or products in the sum of (4.6) except the first one, $u_{i}(P)(z)$, have positive degree in $t$ due to the factors $t^{k}(k \geq 1)$. By using the observations above and keeping track of the degree in $t$, it is easy to see that $u_{i}(P)\left(z+t N_{t}(z)\right)$ can be written as

$$
u_{i}(P)\left(z+t N_{t}(z)\right)=u_{i}(P)(z)+\sum_{j=1}^{\infty} A_{i j}(z) t^{j}
$$

for some $A_{i j}(z) \in \mathcal{U}_{i}(P)(z)(i, j \geq 1)$.

Now, by combining (4.5) and (4.7), we have

$$
\begin{aligned}
\Delta Q_{t}(z) & =\sum_{i=1}^{\infty}\left(u_{i}(P)(z)+\sum_{j=1}^{\infty} A_{i j}(z) t^{j}\right) t^{i-1} \\
& =\sum_{k=1}^{\infty}\left(u_{k}(P)+\sum_{\substack{i+j=k \\
i, j \geq 1}} A_{i j}(z)\right) t^{k-1} \\
& =\sum_{i=1}^{\infty} w_{k}(P)(z) t^{k-1}
\end{aligned}
$$


where, for any $k \geq 1$,

$$
w_{k}(P)(z)=u_{k}(P)+\sum_{\substack{i+j=k \\ i, j \geq 1}} A_{i j}(z) .
$$

Hence we get (4.3). By the fact that $A_{i, j}(z) \in \mathcal{U}_{i}(P)(z)$ for any $i, j \geq 1$, we see that each $A_{i, j}(z)$ in (4.8) lies in $\mathfrak{U}_{k-1}(P)(z)$ since $i \leq k-1$. Therefore (4.4) also holds.

Lemma 4.6. For any $m, k \geq 1$ and $1 \leq l \leq k$, we have

$$
\frac{\partial^{l}}{\partial t^{l}} \Delta^{m} Q_{t}^{m}(z) \equiv \frac{\Delta^{m+l} Q_{t}^{m+l}}{2^{l}(m+1) \cdots(m+l)} \quad \bmod \left(\mathcal{U}_{k}(P), t^{k-l+1}\right) .
$$

Proof. We fix $k \geq 1$ and use the mathematical induction on $l$ to show (4.9) holds for any $m \geq 1$.

By (2.11) with $k=m$ and (4.3), it is easy to see that (4.9) holds for any $m \geq 1$ when $l=1$.

Now we assume that (4.9) holds for any $1 \leq l \leq k_{0}<k$ and consider the case $l=k_{0}+1$. By applying $\frac{\partial}{\partial t}$ to (4.9) with $l=k_{0}$, we have

$$
\frac{\partial^{k_{0}+1} \Delta^{m} Q_{t}^{m}}{\partial t^{k_{0}+1}} \equiv \frac{\frac{\partial}{\partial t} \Delta^{m+k_{0}} Q_{t}^{m+k_{0}}}{2^{k_{0}}(m+1) \cdots\left(m+k_{0}\right)} \quad \bmod \left(\mathcal{U}_{k}(P), t^{k-k_{0}}\right),
$$

while, from (4.9) with $l=1$, we have

$$
\frac{\partial}{\partial t} \Delta^{m+k_{0}} Q_{t}^{m+k_{0}} \equiv \frac{\Delta^{m+k_{0}+1} Q_{t}^{m+k_{0}+1}}{2\left(m+k_{0}+1\right)} \bmod \left(\mathcal{U}_{k}(P), t^{k}\right) .
$$

Since $k-k_{0} \leq k$, hence we also have

$$
\frac{\partial}{\partial t} \Delta^{m+k_{0}} Q_{t}^{m+k_{0}} \equiv \frac{\Delta^{m+k_{0}+1} Q_{t}^{m+k_{0}+1}}{2\left(m+k_{0}+1\right)} \bmod \left(\mathcal{U}_{k}(P), t^{k-k_{0}}\right) .
$$

By combining (4.10) and (4.11), we have

$$
\begin{aligned}
\frac{\partial^{k_{0}+1}}{\partial t^{k_{0}+1}} \Delta^{m} Q_{t}^{m} \equiv \frac{\Delta^{m+k_{0}+1} Q_{t}^{m+k_{0}+1}}{2^{k_{0}+1}(m+1)(m+2) \cdots\left(m+k_{0}+1\right)} & \bmod \left(\mathcal{U}_{k}(P), t^{k-k_{0}}=t^{k-\left(k_{0}+1\right)+1}\right),
\end{aligned}
$$

which is (4.9) for $l=k_{0}+1$.

Now we are ready to prove Theorem 4.1 .

Proof of Theorem 4.1. We use mathematical induction on $k \geq 1$. Since $u_{1}(P)=$ $\Delta P=v_{1}(P)$, the theorem is true for $k=1$.

Now, we assume that $\mathcal{U}_{k}(P)=\mathcal{V}_{k}(P)$ for some $k \geq 1$. By (4.9) with $m=1$ and $l=k$, we have

$$
\frac{\partial^{k}}{\partial t^{k}} \Delta Q_{t} \equiv \frac{\Delta^{k+1} Q_{t}^{k+1}}{2^{k}(k+1) !} \quad \bmod \left(\mathcal{U}_{k}(P), t\right) .
$$


In other words, we have

$$
\left.\left.\frac{\partial^{k}}{\partial t^{k}} \Delta Q_{t}\right|_{t=0} \equiv \frac{\Delta^{k+1} Q_{t}^{k+1}}{2^{k}(k+1) !}\right|_{t=0} \quad \bmod \left(\mathcal{U}_{k}(P)\right) .
$$

On the other hand, by the initial condition in (2.8), we have

$$
\left.\frac{\Delta^{k+1} Q_{t}^{k+1}}{2^{k}(k+1) !}\right|_{t=0}=\frac{\Delta^{k+1} P^{k+1}}{2^{k}(k+1) !}=\frac{v_{k+1}(P)}{2^{k}(k+1) !} .
$$

By (4.3) and (4.4), we have

$$
\left.\frac{\partial^{k}}{\partial t^{k}} \Delta Q_{t}\right|_{t=0}=k ! w_{k+1}(P) \equiv k ! u_{k+1}(P) \quad \bmod \left(\mathcal{U}_{k}(P)\right) .
$$

Therefore, by (4.12) and the two equations above, we have

$$
u_{k+1}(P) \equiv \frac{1}{2^{k} k !(k+1) !} v_{k+1}(P) \quad \bmod \left(\mathcal{U}_{k}(P)\right) .
$$

Since $\mathfrak{U}_{k}(P)=\mathcal{V}_{k}(P)$, we have $\mathfrak{U}_{k+1}(P)=\mathcal{V}_{k+1}(P)$.

\section{Hessian nilpotent polynomials}

In this section, we first derive in Subsection 5.1 a criterion for Hessian nilpotency of homogeneous polynomials by using a fundamental theorem (see Theorem 5.2) of harmonic polynomials. We then give in Subsection 5.2 some examples of HNP (Hessian nilpotent polynomials) and HNS (Hessian nilpotent formal power series).

5.1. A criterion for Hessian nilpotency of homogeneous harmonic polynomials. For any $n \geq 1$, we let $X\left(\mathbb{C}^{n}\right)$ or simply $X$ denote the affine variety defined by $\sum_{i=1}^{n} z_{i}^{2}=0$. For any $d \geq 0$, we denoted by $V_{d}(z)$ the vector space of homogeneous polynomials in $z$ of degree $d \geq 0$. For any $\alpha \in \mathbb{C}^{n}$, we denote by $h_{\alpha}(z)$ the linear function $\langle\alpha, z\rangle$ of $\mathbb{C}^{n}$.

The following identities are almost trivial but very useful for our later arguments, so we formulate them as a lemma without giving proofs.

Lemma 5.1. (a) For any $\alpha \in \mathbb{C}^{n}$ and $m \geq 1$, we have

$$
\operatorname{Hes}\left(h_{\alpha}^{m}\right)(z)=m(m-1) h_{\alpha}^{m-2}(z) \alpha \cdot \alpha^{t} .
$$

(b) For any $\alpha, \beta \in X\left(\mathbb{C}^{n}\right)$ and $m, k \geq 1$, we have

$$
\Delta\left(h_{\alpha}^{m}(z) h_{\beta}^{k}(z)\right)=2 m k\langle\alpha, \beta\rangle h_{\alpha}^{m-1}(z) h_{\beta}^{k-1}(z) .
$$

By Remark 2.4, we know that any $\operatorname{HNS~} P(z)$ is automatically harmonic, i.e. $\Delta P(z)=0$. For harmonic polynomials, we have the following fundamental theorem.

Theorem 5.2. For any homogeneous harmonic polynomial $P(z)$ of degree $d \geq 2$, we have

$$
P(z)=\sum_{i=1}^{k} c_{i} h_{\alpha_{i}}^{d}(z)
$$

for some $c_{i} \in \mathbb{C}$ and $\alpha_{i} \in X\left(\mathbb{C}^{n}\right)(1 \leq i \leq k)$. 
For the proof of this theorem, see, for example, $[\mathrm{H}]$ and $[\mathrm{T}$.

Note that, by replacing $\alpha_{i}$ by $c_{i}^{-\frac{1}{d}} \alpha_{i}(1 \leq i \leq k)$ in (5.3), we see that any homogeneous harmonic polynomial $P(z)$ of degree $d \geq 2$ can be written as

$$
P(z)=\sum_{i=1}^{k} h_{\alpha_{i}}^{d}(z)
$$

for some $\alpha_{i} \in X\left(\mathbb{C}^{n}\right)(1 \leq i \leq k)$.

In the rest of this subsection, we fix a homogeneous harmonic polynomial $P(z) \in$ $V_{d}(z)$ of degree $d \geq 2$ and assume that $P(z)$ is given by (5.4) for some $\alpha_{i} \in X\left(\mathbb{C}^{n}\right)$ $(1 \leq i \leq k)$. We also assume $\left\{h_{\alpha}^{d}(z) \mid \alpha \in \mathbb{C}^{n}\right\}$ are linearly independent in $V_{d}(z)$.

We first define the following matrices associated with $P(z)$ :

$$
\begin{aligned}
& A_{P}=\left(\left\langle\alpha_{i}, \alpha_{j}\right\rangle\right)_{k \times k}, \\
& \Psi_{P}=\left(\left\langle\alpha_{i}, \alpha_{j}\right\rangle h_{\alpha_{j}}^{d-2}(z)\right)_{k \times k} .
\end{aligned}
$$

The main result of this section is the following proposition.

Proposition 5.3. Let $P(z) \in V_{d}(z)$ be given by (5.4). Then, for any $m \geq 1$, we have

$$
\operatorname{Tr} \operatorname{Hes}^{m}(P)=(d(d-1))^{m} \operatorname{Tr} \Psi_{P}^{m} .
$$

In particular, $P(z)$ is $H N$ if and only if the matrix $\Psi_{P}$ is nilpotent.

Proof. First, by (5.4) and (5.1), we can write Hes $(P)$ explicitly as

$$
\operatorname{Hes}(P)=d(d-1) \sum_{i=1}^{k} h_{\alpha_{i}}^{d-2}(z) \alpha_{i} \cdot \alpha_{i}^{t} \text {. }
$$

For any $m \geq 1$, we set $c_{m}=(d(d-1))^{m}$. By (5.8), we have

$$
\begin{aligned}
\operatorname{Hes}^{m}(P) & =c_{m} \sum_{i_{1}, i_{2}, \cdots, i_{m}=1}^{k} h_{\alpha_{i_{1}}}^{d-2}(z) \cdots h_{\alpha_{i_{m}}}^{d-2}(z)\left(\alpha_{i_{1}} \cdot \alpha_{i_{1}}^{t}\right) \cdots\left(\alpha_{i_{m}} \cdot \alpha_{i_{m}}^{t}\right) \\
& =c_{m} \sum_{i_{1}, i_{2}, \cdots, i_{m}=1}^{k} \alpha_{i_{1}}\left(\alpha_{i_{1}}^{t} \cdot \alpha_{i_{2}}\right)\left(\alpha_{i_{2}}^{t} \cdot \alpha_{i_{3}}\right) \cdots\left(\alpha_{i_{m}-1}^{t} \cdot \alpha_{i_{m}}\right) \alpha_{i_{m}}^{t} \\
& \cdot h_{\alpha_{i_{1}}}^{d-2}(z) \cdots h_{\alpha_{i_{m}}}^{d-2}(z) \\
& =c_{m} \sum_{i_{1}, i_{2}, \cdots, i_{m}=1}^{k}\left\langle\alpha_{i_{1}}, \alpha_{i_{2}}\right\rangle\left\langle\alpha_{i_{2}}, \alpha_{i_{3}}\right\rangle \cdots\left\langle\alpha_{i_{m-1}}, \alpha_{i_{m}}\right\rangle \\
& \cdot h_{\alpha_{i_{1}}}^{d-2}(z) \cdots h_{\alpha_{i_{m}}}^{d-2}(z) \alpha_{i_{1}} \cdot \alpha_{i_{m}}^{t} \\
& =c_{m} \sum_{i_{1}, i_{m}=1}^{k}\left(\Psi_{P}^{m-1}\right)_{i_{1}, i_{m}} h_{\alpha_{i_{1}}}^{d-2}(z) \alpha_{i_{1}} \cdot \alpha_{i_{m}}^{t} .
\end{aligned}
$$


By taking the trace of the matrices above, we get

$$
\begin{aligned}
\operatorname{TrHes}^{m}(P) & =c_{m} \sum_{i_{1}, i_{m}=1}^{k}\left(\Psi_{P}^{m-1}\right)_{i_{1}, i_{m}} \operatorname{Tr}\left(\alpha_{i_{1}} \cdot \alpha_{i_{m}}^{t}\right) h_{\alpha_{i_{1}}}^{d-2}(z) \\
& =c_{m} \sum_{i_{1}, i_{m}=1}^{k}\left(\Psi_{P}^{m-1}\right)_{i_{1}, i_{m}}\left\langle\alpha_{i_{m}}, \alpha_{i_{1}}\right\rangle h_{\alpha_{i_{1}}}^{d-2}(z) \\
& =c_{m} \sum_{i_{1}, i_{m}=1}^{k}\left(\Psi_{P}^{m-1}\right)_{i_{1}, i_{m}}\left(\Psi_{P}\right)_{i_{m}, i_{1}} \\
& =c_{m} \operatorname{Tr} \Psi_{P}^{m}
\end{aligned}
$$

Hence, we get (5.7).

Corollary 5.4. Let $P(z) \in V_{d}(z)$ be given by (5.4). Suppose that $P(z)$ is HN. Then the matrix $A_{P}$ must be singular.

Proof. By Proposition 5.3, we have that $\Psi_{P}$ is nilpotent. Therefore, we have

$$
0=\operatorname{det} \Psi_{P}=h_{\alpha_{1}}^{d-2}(z) h_{\alpha_{2}}^{d-2}(z) \cdots h_{\alpha_{k}}^{d-2}(z) \operatorname{det} A_{P} .
$$

Hence, we have $\operatorname{det} A_{P}=0$.

Corollary 5.5. Let $P(z)$ be $H N$ and given by (5.4). Then, for any $2 \leq m \leq d$, we have

$$
\sum_{i, j=1}^{k}\left\langle\alpha_{i}, \alpha_{j}\right\rangle^{m} h_{\alpha_{i}}^{d-m}(z) h_{\alpha_{j}}^{d-m}(z)=0 .
$$

In particular, we have

$$
\sum_{i=1}^{k} P\left(\alpha_{i}\right)=0
$$

Proof. First, note that (5.10) follows directly from (5.9) with $m=d$.

To prove (5.9), we first consider the case $m=2$; the LHS of (5.9) is just $\operatorname{Tr} \Psi_{P}^{2}$ up to a non-zero constant. Hence, by Proposition 5.3, (5.9) holds in this case.

Now consider the case $m>2$. By (5.1), we have

$$
\begin{aligned}
\Delta^{l}\left(h_{\alpha_{i}}^{d-2}(z) h_{\alpha_{j}}^{d-2}(z)\right)=2^{l}(d-2)^{2} & \cdots(d-l-1)^{2} \\
\cdot & \left\langle\alpha_{i}, \alpha_{j}\right\rangle^{l} h_{\alpha_{i}}^{d-2-l}(z) h_{\alpha_{j}}^{d-2-l}(z)
\end{aligned}
$$

for any $1 \leq i, j \leq k$ and $1 \leq l \leq d-2$.

Now by applying $\Delta^{m-2}$ to (5.9) for the case $m=2$ and applying (5.11), it is easy to see that (5.9) holds for any $3 \leq m \leq d-2$.

Actually, by (5.2), the LHS of (5.9) is also $\Delta^{m} P^{2}(z)$ up to a non-zero constant. Hence the corollary above also follows from Theorem 4.3, which implies that $\Delta^{m} P^{2}(z)=0$ for any $m \geq 2$.

One remark is that, by applying similar arguments as above to the equations $\Delta^{m} P^{m}(z)=0$ and $\operatorname{Tr} \Psi_{P}^{m}=0(m \geq 1)$, one can derive more explicit identities satisfied by certain powers of $h_{\alpha_{i}}(z)(1 \leq i \leq k)$. But, in order to keep this paper a certain size, we skip them here. More study on homogeneous HNP's will be given in [Z3]. 
5.2. Some examples of HNP's and HNS. In this subsection, we give some examples of HNS and HNP's.

First, let $\Xi=\left\{\beta_{i} \mid 1 \leq i \leq k\right\}$ be any non-empty subset of $X\left(\mathbb{C}^{n}\right)$ such that $\left\langle\beta_{i}, \beta_{j}\right\rangle=0$ for any $1 \leq i, j \leq k$. For any $d \geq 2$, we set

$$
W_{[\Xi, d]}(z)=\sum_{i=1}^{k} h_{\beta_{i}}^{d}(z) .
$$

For convenience, we also set $W_{[\Xi, d]}(z)=0$ for $\Xi=\emptyset$.

Now let $\widetilde{\Xi}=\left(\Xi_{1}, \Xi_{2}, \cdots, \Xi_{m}, \cdots\right)$ be a sequence of finite subsets of $X\left(\mathbb{C}^{n}\right)$ such that, for any $m_{1}, m_{2} \geq 1$ and any $\beta_{i} \in \Xi_{m_{i}}(i=1,2)$, we have $\left\langle\beta_{1}, \beta_{2}\right\rangle=0$. We set

$$
W_{\cong}(z)=\sum_{m=1}^{\infty} W_{\left[\Xi_{m}, m+1\right]}(z)=\sum_{m=1}^{\infty} \sum_{\beta_{m_{i}} \in \Xi_{m}} h_{\beta_{m_{i}}}^{m+1}(z) .
$$

A more general construction is as follows.

Let $w=\left(w_{1}, w_{2}, \cdots, w_{k}\right)$ be a sequence of commutative variables and $\left(\beta_{1}, \beta_{2}, \cdots, \beta_{k}\right)$ a sequence of elements of $\mathbb{C}^{n}$ with $\left\langle\beta_{i}, \beta_{j}\right\rangle=0(1 \leq i, j \leq k)$. For any formal power series $g(w) \in \mathbb{C}[[w]]$, we define $U_{g}(z) \in \mathbb{C}[[z]]$ by

$$
U_{g}(z)=g\left(h_{\beta_{1}}(z), h_{\beta_{2}}(z), \cdots, h_{\beta_{k}}(z)\right) .
$$

One special case of the construction above is as follows. We introduce new commutative variables $u=\left(u_{1}, u_{2}, \cdots, u_{n}\right)$ and $v=\left(v_{1}, v_{2}, \cdots, v_{n}\right)$. For any $g(z) \in$ $\mathbb{C}[[z]]$, we set

$$
P_{g}(u, v)=g\left(u_{1}+\sqrt{-1} v_{1}, u_{2}+\sqrt{-1} v_{2}, \cdots, u_{n}+\sqrt{-1} v_{n}\right) .
$$

Note that, by setting $w_{i}=z_{i}$ and $\beta_{i} \in X\left(\mathbb{C}^{2 n}\right)$ such that $h_{\alpha_{i}}(u, v)=u_{i}+\sqrt{-1} v_{i}$ $(1 \leq i \leq n)$, we have $U_{g}(u, v)=P_{g}(u, v)$.

The following lemma is easy to check directly by using Lemma 5.1

Lemma 5.6. For any $P(z) \in \mathbb{C}[[z]]$ given by (5.13) and (5.14), we have

$$
\Delta P^{m}(z)=0 .
$$

In particular, $P(z)$ is $H N$.

Note that, by choosing $\widetilde{\Xi}$ in (5.13) and $g(w) \in \mathbb{C}[[w]]$ in (5.14) properly, we can construct many HNS, HNP's and homogeneous HNP's. Unfortunately, all these HNS or HNP's $P(z)$ are of "trivial type" in the sense that their deformed inversion pair $Q_{t}(z)=P(z)$. This can be easily seen from (5.16) and Corollary 3.9 A family of non-trivial HNP's was given in BE1 which was constructed as follows.

Let $H(z)=\left(H_{1}(z), H_{2}(z), \cdots, H_{n}(z)\right) \in \mathbb{C}[z]^{\times n}$. Let $u, v$ be as defined before (5.15) and set

$$
P_{H}(u, v)=\sum_{i=1}^{n} v_{i} H_{i}\left(u_{1}+\sqrt{-1} v_{1}, u_{2}+\sqrt{-1} v_{2}, \cdots, u_{n}+\sqrt{-1} v_{n}\right) .
$$

It was shown in Lemma 1.2 in BE1 that $P_{H}(u, v) \in \mathbb{C}[u, v]$ is $\mathrm{HN}$ if and only if $J H(z)$ is nilpotent. 


\section{More Properties of HN POLYNOMIALS}

In this section, we derive more properties of HNS (Hessian nilpotent formal power series) and HNP's (Hessian nilpotent polynomials). We prove an identity in Proposition 6.1 and an equivalence of certain vanishing properties in Theorem 6.2 of $\left\{\Delta^{k} P^{m}(z) \mid k, m \geq 1\right\}$ for HNS $P(z) \in \mathbb{C}[[z]]$. In Subsection 6.3, we study an isotropic property of $\left\{\Delta^{k} P^{m}(z) \mid k, m \geq 1\right\}$ for homogeneous HNP's $P(z)$.

6.1. An identity of $\mathbf{H N}$ formal power series. Let $P(z) \in \mathbb{C}[[z]]$ be an HNS. For any $k \geq 0$ and $\alpha \geq 1$, we set

$$
u_{k, \alpha}(P)=\frac{\alpha !}{2^{k} k !(k+\alpha) !} \Delta^{k} P^{k+\alpha} .
$$

Proposition 6.1. For any $\alpha, \beta \geq 1$ and $m \geq 0$, we have

$$
u_{m, \alpha+\beta}(P)=\sum_{\substack{k+l=m \\ k, l \geq 0}} u_{k, \alpha}(P) u_{l, \beta}(P) .
$$

More explicitly, we have

$$
\Delta^{m} P^{m+\alpha+\beta}=\left(\begin{array}{c}
\alpha+\beta \\
\alpha
\end{array}\right)^{-1} \sum_{\substack{k+l=m \\
k, l \geq 0}}\left(\begin{array}{c}
m \\
k
\end{array}\right)\left(\begin{array}{c}
m+\alpha+\beta \\
k+\alpha
\end{array}\right)\left(\Delta^{k} P^{k+\alpha}\right)\left(\Delta^{l} P^{l+\beta}\right) .
$$

Proof. First, it is easy to see that (6.3) follows directly from (6.2) and (6.1), so we only need prove (6.2).

By (3.8) and (6.1), we have

$$
Q_{t}^{\gamma}(z)=\sum_{m=0}^{\infty} u_{m, \gamma}(P) t^{m}
$$

for any $\gamma \geq 1$. By comparing the coefficients of $t^{m}$ on both sides of the equation $Q_{t}^{\alpha+\beta}(z)=Q_{t}^{\alpha}(z) Q_{t}^{\beta}(z)$, we see that (6.2) holds.

\subsection{A vanishing property of $\mathbf{H N}$ formal power series.}

Theorem 6.2. For any $H N S P(z) \in \mathbb{C}[[z]]$, the following statements are equivalent:

(1) For any $k \geq 1, \Delta^{m} P^{m+k}=0$ when $m>>0$.

(2) There exists $k_{0} \geq 1, \Delta^{m} P^{m+k_{0}}=0$ when $m>>0$.

(3) $\Delta^{m} P^{m+1}=0$ when $m>>0$.

Proof. (1) $\Rightarrow(2)$ is trivial. To show $(2) \Rightarrow(3)$, we assume that $\Delta^{m} P^{m+k_{0}}=0$ when $m>M_{0}$ for some $M_{0} \geq 1$. For any $m>M_{0}+k_{0}-1$, we have

$$
\Delta^{m} P^{m+1}=\Delta^{k_{0}-1}\left(\Delta^{m-k_{0}+1} P^{\left(m-k_{0}+1\right)+k_{0}}\right)=0 .
$$

Hence (3) holds in this case.

Now we consider $(3) \Rightarrow(1)$. Since $P(z)$ is HN, (3.8) in Theorem 3.4 holds for any $k \geq 1$. In particular, $Q_{t}(z)$ is a polynomial in $t$ with coefficients in $\mathbb{C}[[z]]$ by our assumption of (3). Therefore, for any $k \geq 1, Q_{t}^{k}(z)$ is also a polynomial in $t$ with coefficients in $\mathbb{C}[[z]]$. By (3.8) again, we see that (1) holds. 
We believe that Theorem 7.2 is still true without the Hessian nilpotency condition. Actually, if Conjecture 4.4 is true, it is certainly the case. More precisely, suppose that one of the statements, say (3), of Theorem 7.2 holds for some $P(z) \in \mathbb{C}[[z]]$. Then we have

$$
\Delta^{m+1} P^{m+1}=\Delta\left(\Delta^{m} P^{m+1}\right)=0
$$

when $m>>0$. If Conjecture 4.4 is true, then $P(z)$ is HN. Hence all other statements of Theorem 7.2 also hold.

Later we will show in Theorem 7.2 that the Jacobian conjecture is equivalent to saying that one of the statements in Theorem 6.2 holds for HNP's $P(z)$.

6.3. Isotropic properties of homogeneous $\mathbf{H N}$ polynomials. For any $1 \leq$ $i \leq n$, we set $D_{i}=\frac{\partial}{\partial z_{i}}$ and $D=\left(D_{1}, D_{2}, \cdots, D_{n}\right)$. We define a $\mathbb{C}$-bilinear map $\{\cdot, \cdot\}: \mathbb{C}[z] \times \mathbb{C}[z] \rightarrow \mathbb{C}[z]$ by setting $\{f, g\}=f(D) g(z)$ for any $f, g \in \mathbb{C}[z]$. The $\mathbb{C}$-bilinear map $\{\cdot, \cdot\}$ defined above is closely related to the following commonly used Hermitian inner product of $\mathbb{C}[z]$. See, for example, $[\mathrm{ABR}], \mathrm{H}]$ and $[\mathrm{KR}]$.

$$
\begin{aligned}
(\cdot, \cdot): \mathbb{C}[z] \times \mathbb{C}[z] & \rightarrow \mathbb{C}, \\
(f, \quad g) & \rightarrow(f(D) \bar{g})(0),
\end{aligned}
$$

where $\bar{g}(z)=\sum_{I \in \mathbb{N}^{n}} \bar{a}_{\mathbf{s}} z^{\mathbf{s}}$ if $g(z)=\sum_{I \in \mathbb{N}^{n}} a_{\mathbf{s}} z^{\mathbf{s}}$. In particular, for any homogeneous polynomials $f, g \in \mathbb{C}[z]$ of the same degree, we have $\{f, \bar{g}\}=(f, g)$.

Actually, the Hermitian inner product $(\cdot, \cdot)$ plays a very important role in the study of classical harmonic polynomials (see $\mathrm{ABR}]$ and $[\mathrm{H}]$ ). Due to the connection of $\{\cdot, \cdot\}$ with the Hermitian inner product $(\cdot, \cdot)$ described above, we refer to the properties of HNP's derived in this subsection as certain isotropic properties.

The main result of this subsection is the following theorem.

Theorem 6.3. Let $P(z)$ be a homogeneous HNP of degree $d \geq 3$ and $\mathcal{J}(P)$ the ideal of $\mathbb{C}[z]$ generated by $\sigma^{2}:=\sum_{i=1}^{n} z_{i}^{2}$ and $\frac{\partial P}{\partial z_{i}}(1 \leq i \leq n)$. Then, for any $f(z) \in \mathcal{J}(P)$ and $m \geq 0$, we have

$$
\left\{f, \Delta^{m} P^{m+1}\right\}=f(D) \Delta^{m} P^{m+1}=0 .
$$

To prove this theorem, we first need the following two lemmas.

Lemma 6.4. For any homogeneous polynomial $f(z)$ of degree $k \geq 1$, we have

$$
\begin{aligned}
& \sum_{\substack{i_{1}, i_{2}, \cdots, i_{k}=1 \\
=}}^{n} \frac{\partial^{k} f(z)}{\partial z_{i_{1}} \partial z_{i_{2}} \cdots \partial z_{i_{k}}} \frac{\partial^{k}}{\partial z_{i_{1}} \partial z_{i_{2}} \cdots \partial z_{i_{k}}} \\
= & \sum_{\substack{\mathbf{s} \in \mathbb{N}^{n} \\
|\mathbf{s}|=k}}\left(\begin{array}{l}
k \\
\mathbf{s}
\end{array}\right) \frac{\partial^{k} f}{\partial z^{\mathbf{s}}} \frac{\partial^{k}}{\partial z^{\mathbf{s}}} \\
= & k ! f(D),
\end{aligned}
$$

where $\left(\begin{array}{l}k \\ \mathbf{s}\end{array}\right)=\frac{k !}{s_{1} ! s_{2} ! \cdots s_{n} !}$ for any $\mathbf{s}=\left(s_{1}, s_{2}, \cdots, s_{n}\right) \in \mathbb{N}^{n}$ with $|\mathbf{s}|=k$. 
Proof. Since (6.6) is linear on $f(z)$, we may assume that $f(z)$ is a single monomial, say, $f(z)=z_{1}^{l_{1}} z_{2}^{l_{2}} \cdots z_{n}^{l_{n}}$ with $l_{i} \geq 0$ and $\sum_{i=1}^{n} l_{i}=k$. Now, we consider

$$
\begin{aligned}
& \sum_{i_{1}, i_{2}, \cdots, i_{k}=1}^{n}\left(\frac{\partial^{k}}{\partial z_{i_{1}} \partial z_{i_{2}} \cdots \partial z_{i_{k}}} z_{1}^{l_{1}} z_{2}^{l_{2}} \cdots z_{n}^{l_{n}}\right) \frac{\partial^{k}}{\partial z_{i_{1}} \partial z_{i_{2}} \cdots \partial z_{i_{k}}} \\
& =\left(\frac{k !}{l_{1} ! l_{2} ! \cdots l_{n} !} \frac{\partial^{k}}{\partial z_{1}^{l_{1}} \partial z_{2}^{l_{2}} \cdots \partial z_{n}^{l_{n}}} z_{1}^{l_{1}} z_{2}^{l_{2}} \cdots z_{n}^{l_{n}}\right) \frac{\partial^{k}}{\partial z_{1}^{l_{1}} \partial z_{2}^{l_{2}} \cdots \partial z_{n}^{l_{n}}} \\
& =k !\left(\frac{\partial}{\partial z_{1}}\right)^{l_{1}}\left(\frac{\partial}{\partial z_{2}}\right)^{l_{2}} \cdots\left(\frac{\partial}{\partial z_{n}}\right)^{l_{n}} \\
& =k ! f(D) .
\end{aligned}
$$

Lemma 6.5. For any $f(z), g(z) \in \mathbb{C}[[z]]$ and $l \geq 1$, we have

$$
\begin{aligned}
\Delta^{l}(g f) & =\sum_{\substack{k_{1}+k_{2}+k_{3}=l \\
k_{1}, k_{2}, k_{3} \geq 0}} 2^{k_{2}}\left(\begin{array}{c}
l \\
k_{1}, k_{2}, k_{3}
\end{array}\right) \sum_{i_{1}, i_{2}, \cdots, i_{k_{2}}=1}^{n} \frac{\partial^{k_{2}} \Delta^{k_{1}} g(z)}{\partial z_{i_{1}} \cdots \partial z_{i_{2}}} \frac{\partial^{k_{2}} \Delta^{k_{3}} f(z)}{\partial z_{i_{1}} \cdots \partial z_{i_{k_{2}}}} \\
& =\sum_{\substack{k_{1}+k_{2}+k_{3}=l \\
k_{1}, k_{2}, k_{3} \geq 0}} 2^{k_{2}}\left(\begin{array}{c}
l \\
k_{1}, k_{2}, k_{3}
\end{array}\right) \sum_{\substack{\mathbf{s} \in \mathbb{N}^{n} \\
|\mathbf{s}|=k_{2}}}\left(\begin{array}{c}
k_{2} \\
\mathbf{s}
\end{array}\right) \frac{\partial^{k_{2}} \Delta^{k_{1}} g}{\partial z^{\mathbf{s}}} \frac{\partial^{k_{2}} \Delta^{k_{3}} f}{\partial z^{\mathbf{s}}}
\end{aligned}
$$

where $\left(\begin{array}{c}l \\ k_{1}, k_{2}, k_{3}\end{array}\right)=\frac{l !}{k_{1} ! k_{2} ! k_{3} !}$ for any $k_{1}, k_{2}, k_{3} \geq 0$.

Proof. We use the mathematical induction on $l \geq 1$. When $l=1$, by Leibniz's rule, it is easy to check that

$$
\Delta(g f)(z)=(\Delta g(z)) f(z)+2 \sum_{i=1}^{n} \frac{\partial g(z)}{\partial z_{i}} \frac{\partial f(z)}{\partial z_{i}}+g(z) \Delta f(z)
$$

which is exactly (6.7) with $l=1$.

Now we assume (6.7) holds for $l=l_{0} \geq 0$. By using (6.8) and (6.7) with $l=l_{0}$, we have

$$
\begin{aligned}
& \Delta^{l_{0}+1}(g f)=\Delta\left(\Delta^{l_{0}}(g f)\right) \\
= & \sum_{\substack{k_{1}+k_{2}+k_{3}=l \\
k_{1}, k_{2}, k_{3} \geq 0}} 2^{k_{2}}\left(\begin{array}{c}
l \\
k_{1}, k_{2}, k_{3}
\end{array}\right) \sum_{i_{1}, i_{2}, \cdots, i_{k_{2}}=1}^{n} \Delta\left(\frac{\partial^{k_{2}} \Delta^{k_{1}} g(z)}{\partial z_{i_{1}} \cdots \partial z_{i_{k_{2}}}} \frac{\partial^{k_{2}} \Delta^{k_{3}} f(z)}{\partial z_{i_{1}} \cdots \partial z_{i_{k_{2}}}}\right) \\
= & \sum_{\substack{k_{1}+k_{2}+k_{3}=l \\
k_{1}, k_{2}, k_{3} \geq 0}} 2^{k_{2}}\left(\begin{array}{c}
l \\
k_{1}, k_{2}, k_{3}
\end{array}\right) \sum_{i_{1}, i_{2}, \cdots, i_{k_{2}}=1}^{n} \frac{\partial^{k_{2}} \Delta^{k_{1}+1} g(z)}{\partial z_{i_{1}} \cdots \partial z_{i_{k_{2}}}} \frac{\partial^{k_{2}} \Delta^{k_{3}} f(z)}{\partial z_{i_{1}} \cdots \partial z_{i_{k_{2}}}} \\
+ & \sum_{\substack{k_{1}+k_{2}+k_{3}=l \\
k_{1}, k_{2}, k_{3} \geq 0}} 2^{k_{2}+1}\left(\begin{array}{c}
l \\
k_{1}, k_{2}, k_{3}
\end{array}\right) \sum_{i_{1}, i_{2}, \cdots, i_{k_{2}+1}=1}^{n} \frac{\partial^{k_{2}+1} \Delta^{k_{1}} g(z)}{\partial z_{i_{1}} \cdots \partial z_{i_{k_{2}+1}}} \frac{\partial^{k_{2}+1} \Delta^{k_{3}} f(z)}{\partial z_{i_{1}} \cdots \partial z_{i_{k_{2}+1}}} \\
+ & \sum_{\substack{k_{1}+k_{2}+k_{3}=l \\
k_{1}, k_{2}, k_{3} \geq 0}} 2^{k_{2}}\left(\begin{array}{c}
l \\
k_{1}, k_{2}, k_{3}
\end{array}\right) \sum_{i_{1}, i_{2}, \cdots, i_{k_{2}}=1}^{n} \frac{\partial^{k_{2}} \Delta^{k_{1}} g(z)}{\partial z_{i_{1}} \cdots \partial z_{i_{2}}} \frac{\partial^{k_{2}} \Delta^{k_{3}+1} f(z)}{\partial z_{i_{1}} \cdots \partial z_{i_{k_{2}}}} .
\end{aligned}
$$


By shifting indices and using the convention that $\left(\begin{array}{c}l \\ k_{1}, k_{2}, k_{3}\end{array}\right)=0$ if $k_{1}, k_{2}$ or $k_{3}<0$ :

$$
\begin{aligned}
= & \sum_{\substack{k_{1}+k_{2}+k_{3}=l+1 \\
k_{1}, k_{2}, k_{3} \geq 0}} 2^{k_{2}}\left(\left(\begin{array}{c}
l \\
k_{1}-1, k_{2}, k_{3}
\end{array}\right)+\left(\begin{array}{c}
l \\
k_{1}, k_{2}-1, k_{3}
\end{array}\right)\right. \\
& \left.+\left(\begin{array}{c}
l \\
k_{1}, k_{2}, k_{3}-1
\end{array}\right)\right) \sum_{i_{1}, i_{2}, \cdots, i_{k_{2}}=1}^{n} \frac{\partial^{k_{2}} \Delta^{k_{1}} g(z)}{\partial z_{i_{1}} \cdots \partial z_{i_{2}}} \frac{\partial^{k_{2}} \Delta^{k_{3}} f(z)}{\partial z_{i_{1}} \cdots \partial z_{i_{k_{2}}}} .
\end{aligned}
$$

Hence, we only need to show that

$$
\left(\begin{array}{c}
l+1 \\
k_{1}, k_{2}, k_{3}
\end{array}\right)=\left(\begin{array}{c}
l \\
k_{1}-1, k_{2}, k_{3}
\end{array}\right)+\left(\begin{array}{c}
l \\
k_{1}, k_{2}-1, k_{3}
\end{array}\right)+\left(\begin{array}{c}
l \\
k_{1}, k_{2}, k_{3}-1
\end{array}\right)
$$

for any $k_{1}, k_{2}, k_{3} \geq 0$. But this identity follows directly from the binomal expression of $(x+y+z)^{k}$ for $k=l, l+1$ and the identity

$$
(x+y+z)^{l+1}=(x+y+z)(x+y+z)^{l} .
$$

One immediate consequence of (6.7) is the following corollary.

Corollary 6.6. For $g(z), f(z) \in \mathbb{C}[[z]]$ with $g(z)$ harmonic, we have

$$
\Delta^{l}(g f)=\sum_{k=0}^{l} 2^{k}\left(\begin{array}{l}
l \\
k
\end{array}\right) \sum_{\substack{\mathbf{s} \in \mathbb{N}^{n} \\
|\mathbf{s}|=k}}\left(\begin{array}{l}
k \\
\mathbf{s}
\end{array}\right) \frac{\partial^{k} g}{\partial z^{\mathbf{s}}} \frac{\partial^{k} \Delta^{l-k} f}{\partial z^{\mathbf{s}}} .
$$

Now we are ready to prove Theorem 6.3 .

Proof of Theorem 6.3. Since $P(z)$ is HN, by Theorem 4.3 we know that $\Delta^{m} P^{m}=0$ for any $m \geq 1$. Therefore, we have

$$
\sigma^{2}(D) \Delta^{m} P^{m+1}=\Delta^{m+1} P^{m+1}=0 .
$$

Hence it will be enough to prove the theorem for $f(z)=\frac{\partial P}{\partial z_{i}}(z)$ for any fixed $1 \leq i \leq n$.

Since $d \geq 3$, we have $m+d-1 \geq m+2$ and

$$
\Delta^{m+d-1} P^{m+2}=0 .
$$

By applying $\frac{\partial}{\partial z_{i}}$ to the equation above, we get

$$
\Delta^{m+d-1} \frac{\partial P}{\partial z_{i}} P^{m+1}=\Delta^{m+d-1}\left(f P^{m+1}\right)=0 .
$$

But, on the other hand, by (6.9), we have

$$
=\sum_{k=0}^{\Delta^{m+d-1}\left(f P^{m+1}\right)} 2^{k}\left(\begin{array}{c}
m+d-1 \\
k
\end{array}\right) \sum_{\substack{\mathbf{s} \in \mathbb{N}^{n} \\
|\mathbf{s}|=k}}\left(\begin{array}{l}
k \\
\mathbf{s}
\end{array}\right) \frac{\partial^{k} f}{\partial z^{\mathbf{s}}} \frac{\partial^{k} \Delta^{m+d-1-k} P^{m+1}}{\partial z^{\mathbf{s}}} .
$$

Furthermore, we also have the following equations:

$$
\begin{aligned}
& \frac{\partial^{\mathbf{s}} f}{\partial z^{\mathbf{s}}}=0 \quad \text { for any } \mathbf{s} \in \mathbb{N}^{n} \text { with }|\mathbf{s}|=k>d-1, \\
& \Delta^{a} P^{m+1}=0 \quad \text { for any } a \geq m+1 .
\end{aligned}
$$


Hence all the terms on the RHS of (6.12) except the one with $k=d-1$ are zero. Therefore, by (6.6) in Lemma 6.4, we have

$$
\begin{aligned}
\Delta^{m+d-1}\left(f P^{m+1}\right) & =2^{d-1} \sum_{\substack{\mathbf{s} \in \mathbb{N}^{n} \\
|\mathbf{s}|=d-1}}\left(\begin{array}{c}
d-1 \\
\mathbf{s}
\end{array}\right) \frac{\partial^{d-1} f}{\partial z^{\mathbf{s}}} \frac{\partial^{d-1} \Delta^{m} P^{m+1}}{\partial z^{\mathbf{s}}} \\
& =2^{d-1}(d-1) ! f(D) \Delta^{m} P^{m+1} .
\end{aligned}
$$

Hence, by (6.11) and the equation above, we see that the theorem holds for $f(z)=$ $\frac{\partial P}{\partial z_{i}}(z)(1 \leq i \leq n)$.

Corollary 6.7. Let $P(z)$ be a homogeneous HNP of degree $d \geq 3$ and $Q_{t}(z)$ its deformed inversion pair. Then, for any $k, l \geq 0$ with $k>l$, we have

$$
\left(\Delta^{l} P^{k}\right)(D) Q_{t}(z)=0 \text {. }
$$

More precisely, we have

$$
\left(\Delta^{l} P^{k}\right)(D)\left(\Delta^{m} P^{m+1}(z)\right)=0
$$

for any $k, l \geq 0$ with $k>l$.

In particular, we have

$$
P(D) Q_{t}(z)=0 \text {. }
$$

Proof. First note that (6.15) follows from (6.13) by setting $l=0$ and $k=1$. By (3.8), we see that (6.13) and (6.14) are equivalent to each other. Hence, it is enough to show (6.14). Furthermore, by Theorem 6.3 it will be enough to show that $\Delta^{l} P^{k}(z)$ for any $k, l \geq 0$ with $k>l$ lies in the ideal $\tilde{\mathcal{J}}(P)$ generated by $\frac{\partial P}{\partial z_{i}}(z)$ $(1 \leq i \leq n)$.

By Euler's lemma, we have $P(z)=\frac{1}{d} \sum_{i=1}^{n} z_{i} \frac{\partial P}{\partial z_{i}}(z)$. Hence, for any $k \geq 1$, $P^{k}(z) \in \tilde{\mathcal{J}}(P)$ and (6.14) holds when $l=0$. Now we consider the case $l>0$. Note that $\Delta^{l}$ is a sum of the differential operators of the form $\frac{\partial^{2}}{\partial z_{i_{1}}^{2}} \frac{\partial^{2}}{\partial z_{i_{2}}^{2}} \cdots \frac{\partial^{2}}{\partial z_{i_{l}}^{2}}$ with $1 \leq i_{1}, i_{2}, \cdots, i_{l} \leq n$. When we distribute the $2 l$ derivations $\frac{\partial}{\partial z_{i_{j}}}(1 \leq j \leq l)$ of the differential operator above to $k$ copies $P(z)$ of $P^{k}(z)$, there is always at least one copy $P(z)$ of $P^{k}(z)$ that receives none or one derivation. Otherwise, we would have $2 k \leq 2 l$, which contradicts our condition $k>l$. Since we have already shown $P(z) \in \tilde{\mathcal{J}}(P)$ above, we have $\Delta^{l} P^{k}(z) \in \tilde{\mathcal{J}}(P)$ for any $k, l \geq 0$ with $k>l>0$.

Theorem 6.3 and Corollary 6.7 do not hold for homogeneous HNP's $P(z)$ of degree $d=2$. But, by similar arguments as in the proof of Theorem 6.3 starting from $\Delta^{m+2} P^{m+2}=\Delta^{m+2}\left(P \cdot P^{m+1}\right)=0$ instead of (6.11), one can show the following proposition.

Proposition 6.8. Let $P(z)$ be a homogeneous $H N P$ of degree $d=2$ and $\mathcal{J}(P)$ the ideal of $\mathbb{C}[z]$ generated by $P(z)$ and $\sigma^{2}=\sum_{i=1}^{n} z_{i}^{2}$. Then, for any $f(z) \in \mathcal{J}(P)$ and $m \geq 0$, we have

$$
f(D) \Delta^{m} P^{m+1}=0 .
$$

In particular, we have

$$
P(D) Q_{t}(z)=0
$$




\section{VANISHING CONJECTURES OF HN POLYNOMIALS}

In this section, we propose some conjectures on certain vanishing properties of polynomials $\left\{\Delta^{k} P^{m}(z) \mid m, k \geq 1\right.$ and $\left.m>k\right\}$ for HNP's (Hessian Nilpotent Polynomials) $P(z)$. We also show that these so-called vanishing conjectures are equivalent to the well-known Jacobian conjecture.

Conjecture 7.1 (Vanishing conjecture). For any HN (not necessarily homogeneous) polynomial $P(z)$ of degree $d \geq 2$, its deformed inversion pair $Q_{t}(z)$ is a polynomial in both $t$ and $z$. More precisely, $\Delta^{k} P^{k+1}=0$ when $k>>0$.

Theorem 7.2. The following statements are equivalent:

(1) The vanishing conjecture for homogeneous $H N P$ of degree $d=4$.

(2) The vanishing conjecture for homogeneous HNP of degree $d \geq 2$.

(3) The vanishing conjecture.

(4) The Jacobian conjecture.

Proof. First, it is easy to see that (2) $\Rightarrow(1),(3) \Rightarrow(1)$ and (3) $\Rightarrow(2)$ are trivial. By the gradient reduction in [BE1] and the homogeneous reduction in [BCW], $\mathrm{Y}$ ] on the Jacobian conjecture, we know that the Jacobian conjecture will be true if it is true for polynomial maps $F(z)=z-\nabla P(z)$ with $P(z)$ being homogeneous HNP of degree $d=4$. Therefore, (4) $\Leftrightarrow$ (1) follows directly from (3.8) in Theorem 3.4. Hence we only need to show $(4) \Rightarrow(3)$.

Now we assume the Jacobian conjecture and let $P(z)$ be an HNP of degree $d \geq 2$. Let $F_{t}(z)=z-t \nabla P(z)$ and $G_{t}(z)=z+t \nabla Q_{t}(z)$ be as before. Consider the formal map $U(z, t)=\left(F_{t}(z), t\right)$ from $\mathbb{C}^{n+1} \rightarrow \mathbb{C}^{n+1}$. It is easy to check that the Jacobian of the map $U(z, t)$ with respect to $(z, t)$ is also identically equal to 1 and the formal inverse $V(z, t)$ is given by $V(z, t)=\left(G_{t}(z), t\right)$. Since we have assumed the Jacobian conjecture, $V(z, t)$ must be a polynomial in $(z, t)$. Hence so is $G_{t}(z)$. By (3.8) again, we see that $\Delta^{m} P^{m+1}(z)$ must vanish when $m>>$.

Next, by using the upper bound given in [BCW], Corollary 1.4, for the degrees of inverse maps of polynomial automorphisms of $\mathbb{C}^{n}$, we show that Conjecture 7.1 for homogeneous HNP's can actually be reformulated more precisely as follows.

Conjecture 7.3 (Homogeneous vanishing conjecture). For any homogeneous HNP $P(z)$ of degree $d \geq 2$, we have

(1) $\Delta^{m} P^{m+1}=0$ for any $m>\alpha_{[n, d]}:=\frac{1}{d-2}\left((d-1)^{n-1}-(d-1)\right)$.

(2) For any $k \geq 1, \Delta^{m} P^{m+k}=0$ for any $m>k \alpha_{[n, d]}$.

Proposition 7.4. (a) For any homogeneous $H N P P(z)$ of degree $d \geq 2$, the statements (1) and (2) in Conjecture 7.3 are equivalent.

(b) Conjecture 7.3 for $d \geq 2$ and Conjecture 7.3 for $d=4$ are both equivalent to the Jacobian conjecture.

Proof. (a) First, $(2) \Rightarrow(1)$ is trivial. Now we show that $(1) \Rightarrow(2)$. By (3.8) with $k=1$, we see that the $\operatorname{degree} \operatorname{deg}_{t} Q_{t}(z)$ of $Q_{t}(z)$ with respect to $t$ is less than or equal to $\alpha_{n, d}$, i.e. $\operatorname{deg}_{t} Q_{t}(z) \leq \alpha_{[n, d]}$. Therefore, for any $k \geq 1$, we have $\operatorname{deg}_{t} Q_{t}^{k}(z) \leq k \alpha_{[n, d]}$. By (3.8) again, we see that (2) holds for any $k \geq 1$.

(b) By Theorem 7.2 , it is easy to see that Conjecture 7.3 for $d \geq 2$ or Conjecture 7.3 with $d=4$ implies the Jacobian conjecture. Therefore it will be enough to show that the Jacobian conjecture implies Conjecture 7.3. By Corollary 1.4 in [BCW], 
we know that, for any polynomial automorphism $F(z)$ of $\mathbb{C}^{n}$ with $\operatorname{deg} F(z)=d-1$, $\operatorname{deg} G(z) \leq(d-1)^{n-1}$. By applying this result to the polynomial automorphism $F(z)=z-\nabla P(z)$ and its inverse $G(z)=z-\nabla Q(z)$, we get $\operatorname{deg} Q(z) \leq(d-1)^{n-1}+1$. Furthermore, by (3.8) with $k=1$ and the fact $\operatorname{deg} \Delta^{m} P^{m+1}(z)=(m+1) d-2 m$ $(m \geq 1)$, we get $\Delta^{m} P^{m+1}(z)=0$ if

$$
(m+1) d-2 m>(d-1)^{n-1}+1 .
$$

By separating $m$ from the inequality above, we get $\Delta^{m} P^{m+1}(z)=0$ whenever $m>\alpha_{[n, d]}$.

Finally, by translating certain known results on the Jacobian conjecture, we know that the vanishing conjectures, Conjectures 7.1 or 7.3 are true for the following cases:

- S. Wang Wa proved that the Jacobian conjecture holds for any polynomial map $F(z)$ of $\operatorname{deg} F(z) \leq 2$. Hence Conjectures 7.1 and 7.3 hold for any $\operatorname{HNP} P(z)$ of degree $d \leq 3$.

- For any symmetric polynomial map $F(z)=z-H(z)$ with $o(H(z)) \geq 2$ and $J H(z)$ nilpotent, A. van den Essen and S. Washburn [EW] showed that the Jacobian conjecture holds when $n \leq 4$ and $H(z)$ is homogeneous. Later, M. de Bondt and A. van den Essen [BE2] BE4 further proved that the Jacobian conjecture holds either $n \leq 4$ without $H(z)$ being homogeneous, or $n=5$ with $H(z)$ being homogeneous. (For an exposition discussion on these results, see [BE5].) From the results above, we see that Conjecture 7.1 has an affirmative answer when $n \leq 4$, and Conjecture 7.3 is true when $n \leq 5$.

- By Theorem 4.1 in [EW] and similar arguments there, it is easy to show that the only HNP's (not necessarily homogeneous) $P(z) \in \mathbb{R}[z](o(P(z)) \geq 2$ ) with real coefficients are $P(z)=0$. Hence, Conjectures 7.1 and 7.3 hold trivially in this case.

- Recently, D. Wright [Wr1] showed that the Jacobian conjecture holds for any symmetric polynomial map $F(z)=z-H(z)$ with $H(z)$ homogeneous and $\mathrm{JH}^{3}(z)=0$. Hence Conjecture 7.3 holds for any homogeneous HNP $P(z)$ with $\operatorname{Hes}^{3}(P(z))=0$.

Furthermore, by Corollary 3.9, we also know that Conjecture 7.1 holds for any HNP $P(z)$ such that $\Delta P^{2}(z)=0$. In particular, it is true for HNP's constructed by (5.13) and (5.14).

\section{ACKNOWLEDGMENT}

The author is very grateful to Professor Arno van den Essen who has carefully read through the first preprint of this paper and pointed out many misprints and several mistakes. Great thanks also go to Professor David Wright for personal communications, especially for informing the author of some of his own recent results. The author also would like to thank Professor Mohan Kumar for personal communications.

\section{ReFERENCES}

[Ar] V. I. Arnold, Mathematical Methods of Classical Mechanics, Springer-Verlag New York, 1978. MR0690288 (57:14033b) 
[ABR] S. Axler, P. Bourdon and W. Ramey, Harmonic function theory. Second edition. Graduate Texts in Mathematics, 137. Springer-Verlag, New York, 2001. MR.1805196 (2001j:31001)

[BCW] H. Bass, E. Connell and D. Wright, The Jacobian conjecture, reduction of degree and formal expansion of the inverse. Bull. Amer. Math. Soc. 7 (1982), 287-330. MR0663785 (83k:14028)

[BE1] M. de Bondt and A. van den Essen, A Reduction of the Jacobian Conjecture to the Symmetric Case, Report No. 0308, University of Nijmegen, June, 2003. Proc. of the AMS. 133 (2005), no. 8, 2201-2205 MR2138860(2006a:14107)

[BE2] M. de Bondt and A. van den Essen, Nilpotent Symmetric Jacobian Matrices and the Jacobian Conjecture, Report No. 0307, University of Nijmegen, June, 2003. J. Pure and Appl. Alg. 193 (2004), no. 1-3, 61-70. MR2076378(2005c:14087)

[BE3] M. de Bondt and A. van den Essen, Singular Hessians, Report No. 0317, University of Nijmegen, October, 2003. J. Algebra 282 (2004), no. 1, 195-204 MR2095579 (2005i:13031)

[BE4] M. de Bondt and A. van den Essen, Nilpotent Symmetric Jacobian Matrices and the Jacobian Conjecture II, Report No. 0318, University of Nijmegen, November, 2003. J. Pure and Appl. Alg. 196 (2005), no. 2-3, 135-148. MR.2110519 (2005k:14130)

[BE5] M. de Bondt and A. van den Essen, Hesse and the Jacobian Conjecture, Report No. 0321, University of Nijmegen, December, 2003.

[E] A. van den Essen, Polynomial automorphisms and the Jacobian conjecture. Progress in Mathematics, 190. Birkhäuser Verlag, Basel, 2000. MR1790619 (2001j:14082)

[EW] A. van den Essen and S. Washburn, The Jacobian conjecture for symmetric Jacobian matrices, J. Pure Appl. Algebra 189 (2004), no. 1-3, 123-133. MR2038568(2004m:14133)

$[\mathrm{H}] \quad$ H. Henryk, Topics in classical automorphic forms, Graduate Studies in Mathematics, 17. American Mathematical Society, Providence, RI, 1997. [MR1474964]. MR1474964 (98e:11051)

[Ke] O. H. Keller, Ganze Gremona-Transformation, Monats. Math. Physik 47 (1939), 299-306.

[KR] V. Kac and A. K. Raina, Bombay Lectures on Highest Weight Representations of Infinite Dimensional Lie Algebras, Advanced Series in Mathematical Physics, 2. World Scientific Publishing Co., Inc., Teaneck, NJ, 1987. MR1021978 (90k:17013)

[M] G. Meng, Legendre Transform, Hessian Conjecture and Tree Formula, math-ph/0308035.

[R] J. Rauch, Partial Differential Equations, Springer-Verlag, New York, 1991. MR.1223093 (94e:35002)

[S] S. Smale, Mathematical Problems for the Next Century, Math. Intelligencer 20, No. 2, 7-15, 1998. MR.1631413 (99h:01033).

[T] M. Takeuchi, Modern spherical functions, Translations of Mathematical Monographs, 135. American Mathematical Society, Providence, RI, 1994. MR1280269 (96d:22009)

[Wa] S. Wang, A Jacobian criterion for separability, J. Algebra 65 (1980), 453-494. MR $0585736(83 \mathrm{e}: 14010)$

[Wr1] D. Wright, Ideal Membership Questions Relating to the Jacobian Conjecture. To appear.

[Wr2] D. Wright, The Jacobian Conjecture: Ideal Membership Questions and Recent Advances, To appear.

[Wr3] D. Wright, Personal communications.

[Y] A. V. Jagžev, On a problem of O.-H. Keller. (Russian) Sibirsk. Mat. Zh. 21 (1980), no. 5, 141-150, 191. MR0592226 (82e:14020)

[Z1] W. Zhao, Recurrent Inversion Formulas, math. CV/0305162.

[Z2] W. Zhao, Inversion Problem, Legendre Transform and the Inviscid Burgers' Equation, J. Pure Appl. Algebra 199 (2005), no.1-3, 299-317. MR2134306 (2006b:14109)

[Z3] W. Zhao, Some Properties and Open Problems of Hessian Nilpotent polynomials, In preparation.

Department of Mathematics, Illinois State University, Normal, Illinois 61790-4520

E-mail address: wzhao@ilstu.edu 